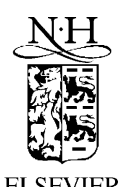

Nuclear Physics B 597 (2001) 197-227

www.elsevier.nl/locate/npe

\title{
Paramagnetic dominance, the sign of the beta function and UV/IR mixing in non-commutative $U(1)$
}

\author{
C.P. Martín, F. Ruiz Ruiz \\ Departamento de Física Teórica I, Facultad de Ciencias Físicas, \\ Universidad Complutense de Madrid, 28040 Madrid, Spain
}

Received 18 July 2000; accepted 4 December 2000

\begin{abstract}
$U(1)$ gauge theory on non-commutative Minkowski spacetime in the Feynman-'t Hooft background gauge is studied. In particular, UV divergences and non-commutative IR divergent contributions to the two, three and four-point functions are explicitly computed at one loop. We show that the negative sign of the beta function results from paramagnetism - producing UV charge anti-screening - prevailing over diamagnetism — giving rise to UV charge screening. This dominance in the field theory setting corresponds to tachyon magnification dominance in the string theory framework. Our calculations provide an explicit realization of UV/IR mixing and lead to an IR renormalization of the coupling constant, where now paramagnetic contributions produce screening and diamagnetic contributions anti-screening. @ 2001 Elsevier Science B.V. All rights reserved.
\end{abstract}

PACS: 11.15.-q; 11.30.Pb; 11.10.Gh

Keywords: Non-commutative $U(1)$ gauge theory; Paramagnetism; Diamagnetism; Beta function

\section{Introduction}

It is a long established fact that the negative sign of the one-loop beta function of QCD can be beautifully explained as the result of a competition between the paramagnetic and diamagnetic contributions to the bare coupling constant obtained from integrating out very high-energy degrees of freedom. Indeed, the paramagnetic contribution, which anti-screens the charge, prevails over the diamagnetic contribution, which screens the charge [1,2]. This dominance can be explicitly computed by evaluating the high-energy quantum fluctuations around a classical field configuration [3], which in turn can be done in a very elegant way using the background field method [4].

E-mail addresses: carmelo@elbereth.fis.ucm.es (C.P. Martín), t63@aeneas.fis.ucm.es (F. Ruiz Ruiz). 
The vacuum-to-vacuum amplitude in the presence of an $S U(N)$ background field $B_{\mu}$ is given by the partition function $Z[B]$, which up to one loop, reads in the Feynman-'t Hooft background field gauge

$$
Z[B]=e^{i S_{\mathrm{cl}}[B]} \int \mathcal{D} Q \mathcal{D} c \mathcal{D} \bar{c} \exp \left\{\frac{i}{2 g_{0}^{2}}\left[S^{\text {diam }}+S^{\text {param }}\right]\right\},
$$

where

$$
\begin{aligned}
S^{\text {diam }} & =-\int d^{D} x \operatorname{Tr}\left\{\left(D_{\mu}[B] Q_{\nu}\right)\left(D^{\mu}[B] Q^{\nu}\right)-2 \bar{c} D_{\mu}[B] D^{\mu}[B] c\right\}, \\
S^{\text {param }} & =2 \int d^{D} x \operatorname{Tr}\left\{F^{\mu \nu}[B] Q^{\sigma}\left(S_{\mu \nu}\right)_{\sigma \rho} Q^{\rho}\right\} .
\end{aligned}
$$

Here $D[B]_{\mu}=\partial_{\mu}-i\left[B_{\mu},\right]$ and $F_{\mu \nu}[B]$ denote the covariant derivative and the field strength for the background field $B_{\mu}$, the $S U(N)$ gauge field $Q_{\mu}$ describes the quantum fluctuations about the background and $\left(S_{\mu \nu}\right)_{\sigma \rho}$ are the generators of the Lorentz group in the spin one representation, i.e.,

$$
\left(S_{\mu \nu}\right)_{\sigma \rho}=i\left(\eta_{\mu \sigma} \eta_{\nu \rho}-\eta_{\mu \rho} \eta_{\nu \sigma}\right) .
$$

The high-momentum modes contributing to the path integral $Z[B]$ yield a logarithmic UV divergence. This divergence shows in dimensional regularization as a pole in $\varepsilon=0$, with $D=4+2 \varepsilon$. As a matter of fact, introducing dimensional regularization and integrating over $Q_{\mu}$, one obtains

$$
\begin{aligned}
\Gamma[B]= & \frac{1}{i} \ln Z[B] \\
= & -\frac{1}{2}\left(\frac{1}{g_{0}^{2}}+\frac{a^{\text {diam }}}{\varepsilon}+\frac{a^{\text {param }}}{\varepsilon}\right) \int d^{D} x \operatorname{Tr}\left(F^{\mu \nu}[B] F_{\mu \nu}[B]\right) \\
& +\Gamma_{\text {finite }}[B],
\end{aligned}
$$

where $^{1}$

$$
a^{\text {diam }}=\frac{N}{16 \pi^{2}}\left[\frac{1}{3}\right], \quad a^{\text {param }}=\frac{N}{16 \pi^{2}}[-4]
$$

and $\Gamma_{\text {finite }}[B]$ collects all finite contributions as $D \rightarrow 4$. The origin of the coefficients $a^{\text {diam }}$ and $a^{\text {param }}$ can be explained as follows. The orbital motion of the charged quanta with only two polarizations ( $D-2$ in $D$ spacetime dimensions) of the gluon field $Q_{\mu}$ in the background $B_{\mu}$ yields "diamagnetism". This motion is described by $S^{\text {diam }}$ above and its contribution to the r.h.s. of Eq. (1.3) has a singular part coming from high-energy quanta which is given by $a^{\mathrm{diam}} / \varepsilon$. On the other hand, $S^{\text {paran }}$ involves the spin current $Q^{\sigma}\left(S_{\mu \nu}\right)_{\sigma \rho} Q^{\rho}$ and describes the coupling between the spin of the gluon field $Q_{\mu}$ and the background $B_{\mu}$. This coupling gives rise to "paramagnetism" and the contribution to $\Gamma[B]$ of the high-momentum quanta involved in it is $a^{\text {param }} / \varepsilon$. One next defines the renormalized coupling constant or renormalized charge $g(\mu)$ in the MS scheme at one loop as usual:

\footnotetext{
${ }^{1}$ In this paper, to emphasize the value of diamagnetic and paramagnetic contributions, we will enclose the relevant factors in square brackets, as in Eq. (1.4).
} 


$$
\frac{1}{g_{0}^{2}}+\frac{a^{\text {diam }}}{\varepsilon}+\frac{a^{\text {param }}}{\varepsilon}=\frac{1}{g^{2}(\mu) \mu^{2 \varepsilon}} .
$$

Hence, the one-loop beta function $\beta\left(g^{2}\right)$ reads for $D=4$

$$
\beta\left(g^{2}\right)=\mu \frac{d g^{2}}{d \mu}=2 g^{2}(\mu)\left(a^{\text {diam }}+a^{\text {param }}\right)=\frac{N}{16 \pi^{2}} g^{4}(\mu)\left[-\frac{22}{3}\right] .
$$

From this equation for the beta function one can draw the following conclusions. First, the coefficient $a^{\text {diam }}$ being positive implies that its effect is to make the charge $g^{2}(\mu=\Lambda)$ decrease with the momentum scale $\Lambda$. This is the charge screening effect due to the orbital motion of the two physical polarizations of the quanta $Q_{\mu}$ in the field $B_{\mu}$. Secondly, since $a^{\text {param }}$ is negative, its effect is to make $g^{2}(\mu=\Lambda)$ grow as $\Lambda$ decreases. This is called antiscreening of the charge and it is due to the interaction of the spin with the field $B_{\mu}$. And thirdly, the inequalities $\left|a^{\text {param }}\right|>a^{\text {diam }}$ and $a^{\text {param }}<0$ explain quantitatively the negative sign of the beta function of the theory, hence, that the charge goes to zero as $\Lambda$ goes to infinity (asymptotic freedom).

It is already a year since it has been shown $[7,8]$ that $U(1)$ gauge theory on noncommutative $\mathbb{R}^{4}$ has an UV divergent behaviour at one loop very similar to that of conventional Yang-Mills theory (see [9] for the $U(N)$ case). In fact, the one-loop beta function is also negative [7], which leads to asymptotic freedom. By contrast, as discovered in Ref. [10], the IR behaviour of non-commutative $U(1)$ gauge theory presents completely novel features $[11,12]$. Indeed, the renormalization of UV divergences induce IR divergences, thus yielding a relation between UV and IR divergences which has been interpreted as a sort of UV/IR duality. This duality seems not to be an artifact of perturbation theory [13], since it has been re-obtained by defining the field theory as the infinite tension limit of the appropriate open bosonic string theory on a magnetic $B$-field $[14,15]$.

The purpose of this paper is two-fold. First, to investigate if, in analogy with conventional Yang-Mills theory, the one-loop beta function of $U(1)$ gauge theory on non-commutative Minkowski space can be understood as a dominance of paramagnetism over diamagnetism. And secondly, to study through explicit computations UV/IR mixing from the point of view of paramagnetism versus diamagnetism. The paper is organized as follows. In Section 2, we will explicitly compute the two-point function and show that the one-loop beta function of $U(1)$ gauge theory on non-commutative Minkowski space has a paramagnetic contribution, producing anti-screening of the charge, and a diamagnetic contribution, giving rise to screening of the charge. We will see that the paramagnetic contribution dominates, thus providing a negative beta function. Furthermore, we will take advantage of the computations in Ref. [15] to show that these paramagnetic and diamagnetic contributions can be given a stringy interpretation as tachyon magnification and zero mode contributions, in the same sense as for conventional Yang-Mills theory [5]. In Sections 3 and 4 we will calculate the UV divergent terms and the leading noncommutative IR terms of the three and four-point functions. It will turn out that the UV/IR mixing occurs for paramagnetic and diamagnetic logarithmic contributions separately. Section 5 shows that the leading non-commutative IR terms computed in Sections 3 and 4 
are consistent with the BRS identities and Section 6 summarizes the conclusions. We will argue there how our results lead to an IR renormalization of the coupling constant.

\section{The vacuum polarization tensor}

Non-commutative Minkowski spacetime is defined by the algebra generated by the operators $X^{\mu}(\mu=0, \ldots, D-1)$ subject to the commutation relations

$$
\left[X^{\mu}, X^{\nu}\right]=i \theta^{\mu \nu}
$$

where $\theta^{\mu \nu}$ is an anti-symmetric real matrix and contraction of indices is performed with the Minkowski metric. We shall take $\theta^{\mu \nu}$ to be "magnetic", i.e., $\theta^{0 i}=0$ for $i=1,2,3$, since in this case the field theory exists as the zero slope limit of a string theory in a magnetic background. If $\theta^{\mu \nu}$ is "electric", the field theory does not exist as the zero slope limit of a string theory [16] and does not make sense on its own since it does not preserve unitarity [17]. Without loss of generality, we will take $\theta^{\mu \nu}$ to vanish for all $\mu$ and $\nu$, except for $\mu, v=1,2$, for which we write

$$
\theta^{12}=-\theta^{21} \equiv \theta \text {. }
$$

The classical action of $U(1)$ gauge theory on non-commutative Minkowski spacetime is given by

$$
S_{\text {class }}[A]=-\frac{1}{4 g^{2}} \int d^{D} x\left(F^{\mu \nu} \star F_{\mu \nu}\right)(x),
$$

where

$$
\begin{gathered}
F_{\mu \nu}(x)=\partial_{\mu} A_{\nu}(x)-\partial_{\nu} A_{\mu}(x)-i\left[A_{\mu}, A_{\nu}\right](x), \\
{\left[A_{\mu}, A_{\nu}\right]=\left(A_{\mu} \star A_{\nu}-A_{\nu} \star A_{\mu}\right)(x)}
\end{gathered}
$$

is the field strength and the symbol $\star$ stands for the Moyal product of functions

$$
(f \star g)(x)=f(x) \exp \left\{\frac{i}{2} \theta^{\mu \nu} \overleftarrow{\partial}_{\mu} \vec{\partial}_{\nu}\right\} g(x)
$$

The classical theory is invariant under non-commutative $U(1)$ gauge transformations, which in infinitesimal form have the form

$$
\delta_{\omega} A_{\mu}(x)=D_{\mu}[A] \omega=\partial_{\mu} \omega(x)-i\left[A_{\mu}, \omega\right](x),
$$

the commutator being defined with regard to the Moyal product.

To quantize the theory around a background field configuration, say $B_{\mu}(x)$, we shall use the background field method in the Feynman-'t Hooft gauge. To this end, we write the gauge field $A_{\mu}$ as the sum of the the background $B_{\mu}$ and the quantum fluctuation $Q_{\mu}$ about it, $A_{\mu}=B_{\mu}+Q_{\mu}$. The tree-level action $S$ then becomes

$$
S=S_{\text {class }}[B+Q]+S_{\mathrm{gf}}+S_{\mathrm{gh}},
$$

where the gauge fixing and ghost terms have the form 


$$
\begin{aligned}
& S_{\mathrm{gf}}=-\frac{1}{2} \int d^{D} x D_{\mu}[B] Q^{\mu} \star D_{\nu}[B] Q^{\nu}, \\
& S_{\mathrm{gh}}=\int d^{D} x \bar{c} \star D_{\mu}[B+Q] D^{\mu}[B] c .
\end{aligned}
$$

The fields $c$ and $\bar{c}$ are the ghost fields and the commutators in the covariant derivatives

$$
D_{\mu}[B+Q]=\partial_{\mu}-i\left[B_{\mu}+Q_{\mu},\right], \quad D_{\mu}(B)=\partial_{\mu}-i\left[B_{\mu},\right]
$$

are defined with regard to the Moyal product. Some straightforward manipulations give for the partition function $Z[B]$ of the theory up to one loop in the $U(1)$ background field $B_{\mu}$ the expression

$$
Z[B]=e^{i S_{\mathrm{cl}}[B]} \int \mathcal{D} Q \mathcal{D} c \mathcal{D} \bar{c} \exp \left[\frac{i}{2 g_{0}^{2}}\left(S^{\mathrm{diam}}+S^{\mathrm{param}}\right)\right],
$$

with

$$
\begin{aligned}
S^{\text {diam }} & =-\int d^{D} x\left(D_{\mu}[B] Q_{\nu} \star D^{\mu}[B] Q^{\nu}-2 \bar{c} \star D_{\mu}[B] D^{\mu}[B] c\right), \\
S^{\text {param }} & =2 \int d^{D} x F^{\mu \nu}[B] \star Q^{\sigma} \star\left(S_{\mu \nu}\right)_{\sigma \rho} Q^{\rho},
\end{aligned}
$$

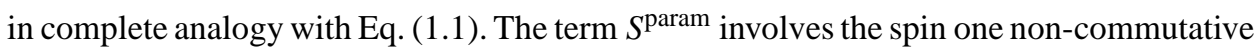
current $Q^{\sigma} \star\left(S_{\mu \nu}\right)_{\sigma \rho} Q^{\rho}$ and describes the coupling of the spin to the background field $B_{\mu}$. This term thus gives rise to non-commutative Pauli "paramagnetism". In turn, the functional $S^{\text {diam }}$ is the classical action for the motion in the field $B_{\mu}$ of the $D-2$ physical degrees of freedom of the field $Q_{\mu}$. Indeed,

$$
\int \mathcal{D} Q \mathcal{D} c \mathcal{D} \bar{c} \exp \left[\frac{i}{2 g_{0}^{2}} S^{\text {diam }}\right]=\left(\operatorname{det}^{-1 / 2} D^{2}[B]\right)^{D-2} .
$$

We shall then say that $S^{\text {diam }}$ gives rise to non-commutative Landau "diamagnetism". From $S^{\text {diam }}$ and $S^{\text {param }}$ one readily extracts the Feynman rules needed for one-loop perturbative computations. We have collected them in Fig. 1, where we have used the notation

$$
q \wedge p=\theta^{\mu v} q_{\mu} p_{v}
$$

Vertices coming from $S^{\text {diam }}$ and $S^{\text {param }}$ will be called diamagnetic and paramagnetic, respectively.

In this section we compute up to one loop the vacuum polarization tensor $\Pi_{\mu \nu}(p)$, defined as

$$
i \Pi_{\mu \nu}(x, y)=\left.\frac{\delta^{2} \Gamma[B]}{\delta B_{\mu}(x) \delta B_{\nu}(y)}\right|_{B=0}=\int \frac{d^{D} p}{(2 \pi)^{D}} e^{-i p \cdot(x-y)} i \Pi_{\mu \nu}(p),
$$

where $i \Gamma[B]=\ln Z[B]$. According to the nature of their vertices, the one-loop Feynman diagrams contributing to $\Pi_{\mu \nu}(p)$ fall into three categories: diagrams with only diamagnetic vertices, diagrams with only paramagnetic vertices and diagrams with both diamagnetic and paramagnetic vertices. The contributions to the vacuum polarization tensor coming 

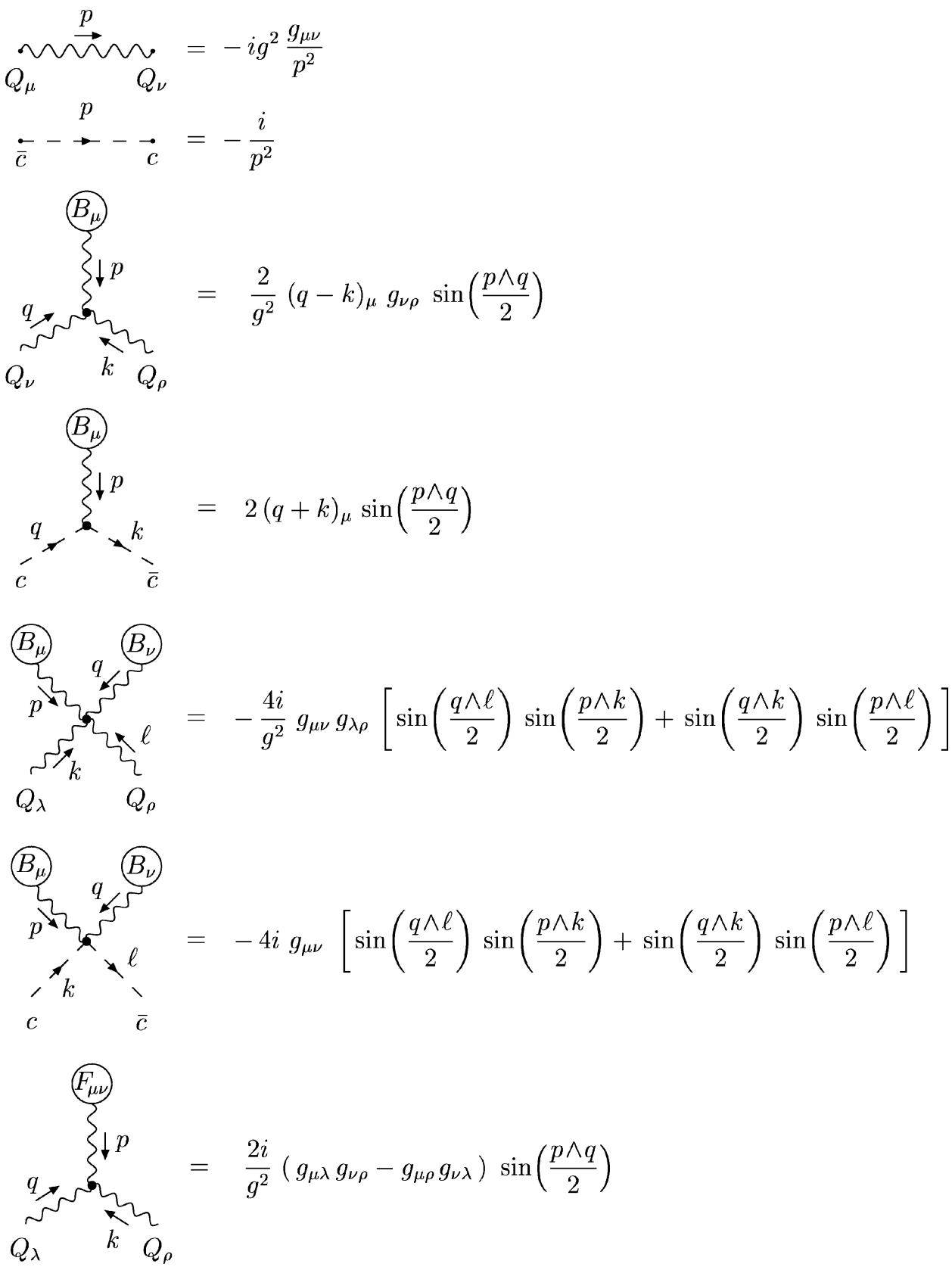

Fig. 1. Feynman rules of $U(1)$ gauge theory on non-commutative Minkowski spacetime in the Feynman background gauge. The last vertex is the only one of paramagnetic type. 
from these three categories will be denoted by $\Pi_{\mu \nu}^{\text {diam }}(p), \Pi_{\mu \nu}^{\mathrm{param}}(p)$ and $\Pi_{\mu \nu}^{\mathrm{mixed}}(p)$, so we write

$$
i \Pi_{\mu \nu}(p)=i \Pi_{\mu \nu}^{\mathrm{diam}}(p)+i \Pi_{\mu \nu}^{\mathrm{param}}(p)+i \Pi_{\mu \nu}^{\operatorname{mixed}}(p) .
$$

It is very easy to see that there is only one diagram contributing to $i \Pi_{\mu \nu}^{\operatorname{mixed}}(p)$, namely that depicted in Fig. 2. Upon performing some algebra in the integrand, the corresponding Feynman integral takes the form

$$
2 i \int \frac{d^{D} q}{(2 \pi)^{D}}\left(\eta_{\mu \nu}-\eta_{\mu \nu}\right) \sin ^{2}\left(\frac{q \wedge p}{2}\right) \frac{(2 q+p)_{\rho}}{q^{2}(q+p)^{2}}=0 .
$$

Hence

$$
\Pi_{\mu \nu}^{\operatorname{mixed}}(p)=0
$$

We are thus left with the diamagnetic and paramagnetic contributions.

The one-loop contribution to $i \Pi_{\mu \nu}^{\operatorname{diam}}(p)$ is given by the sum of the diagrams in Fig. 3, which using the Feynman rules reads

$$
i \Pi_{\mu \nu}^{\operatorname{diam}}(p)=[D-2] \int \frac{d^{D} q}{(2 \pi)^{D}} 2 \sin ^{2}\left(\frac{q \wedge p}{2}\right)\left[\frac{(p+2 q)_{\mu}(p+2 q)_{\nu}}{q^{2}(p+q)^{2}}-2 \frac{\eta_{\mu \nu}}{q^{2}}\right] .
$$

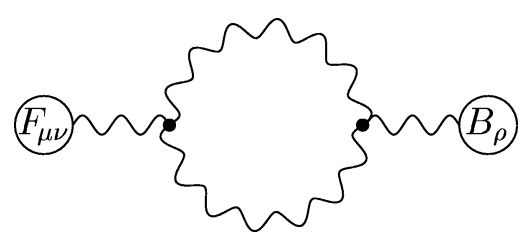

Fig. 2. One-loop Feynman diagram mixing diamagnetic and paramagnetic vertices contributing to the vacuum polarization tensor.
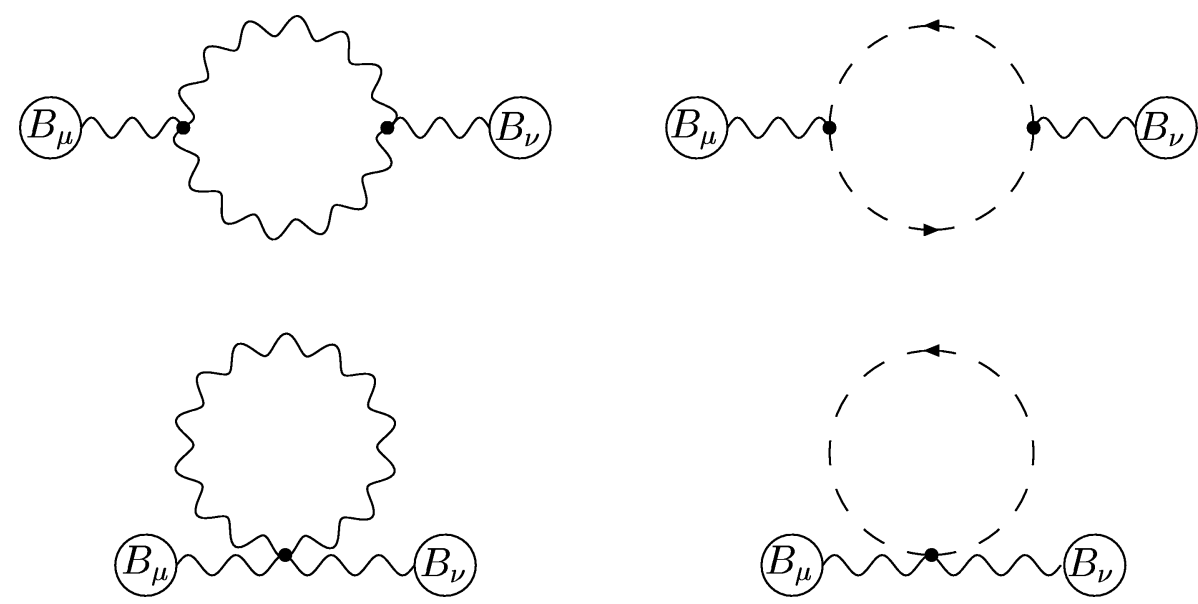

Fig. 3. One-loop diamagnetic Feynman diagrams contributing to the vacuum polarization tensor. The diagrams include planar and non-planar terms. 
The $D$ in the factor $[D-2]$ in front of the integral comes from the diagrams with photons flowing around the loop, whereas the diagrams with ghost loops yield the contribution -2 . Hence the effective one-loop contribution to $i \Pi_{\mu \nu}^{\text {diam }}(p)$ corresponds to $D-2$ scalar fields (transforming in the adjoint representation of the gauge group) moving in the field $B_{\mu}$, in agreement with the discussion following Eqs. (2.3) and (2.4) above. To keep to a minimum the number of diagrams to draw, we have not considered planar and nonplanar diagrams separately. In fact, each of our diagrams is the sum of a planar and a nonplanar contribution. To disentangle these contributions from one another, it is enough to use the identity $4 \sin ^{2}(q \wedge p / 2)=2-e^{i q \wedge p}-e^{-i q \wedge p}$. This identity gives, upon substitution in (2.8), a $\theta^{\mu \nu}$-independent integral, which defines the planar contribution $i \Pi^{\text {diam, } \mathrm{P}}(p)$, and a $\theta^{\mu \nu}$-dependent integral, which constitutes the non-planar contribution $i \Pi^{\text {diam, NP }}(p)$ :

$$
i \Pi_{\mu \nu}^{\operatorname{diam}}(p)=i \Pi_{\mu \nu}^{\mathrm{diam}, \mathrm{P}}(p)+i \Pi_{\mu \nu}^{\mathrm{diam}, \mathrm{NP}}(p) .
$$

After introducing Schwinger parameters, performing the momenta integrals in dimensional regularization and integrating by parts to factorize out the transverse tensor $p^{2} \eta_{\mu \nu}-p_{\mu} p_{\nu}$, we obtain

$$
\begin{aligned}
& i \Pi_{\mu \nu}^{\operatorname{diam}, \mathrm{P}}(p) \\
& \quad=-i \frac{[D-2]}{(4 \pi)^{D / 2}}\left(p^{2} \eta_{\mu \nu}-p_{\mu} p_{\nu}\right) \int_{0}^{1} d x(1-2 x)^{2} \int_{0}^{\infty} d t t^{1-D / 2} e^{-p^{2} t x(1-x)}
\end{aligned}
$$

and

$$
\begin{aligned}
& i \Pi_{\mu \nu}^{\text {diam, }, \mathrm{PP}}(p) \\
& =i \frac{[D-2]}{(4 \pi)^{D / 2}}\left[\left(p^{2} \eta_{\mu \nu}-p_{\mu} p_{\nu}\right) \int_{0}^{1} d x(1-2 x)^{2} \int_{0}^{\infty} d t t^{1-D / 2} e^{-p^{2} t x(1-x)-\tilde{p}^{2} / 4 t}\right. \\
& \left.\quad+\tilde{p}_{\mu} \tilde{p}_{\nu} \int_{0}^{1} d x \int_{0}^{\infty} d t t^{-1-D / 2} e^{-p^{2} t x(1-x)-\tilde{p}^{2} / 4 t}\right]
\end{aligned}
$$

where

$$
\tilde{p}^{\mu}=\theta^{\mu \nu} p_{\nu}, \quad \tilde{p}^{2}=p_{\mu} \theta^{\mu \rho} \theta_{\rho}^{\nu} p_{\nu} .
$$

It is well known [18] that when a Feynman amplitude is expressed in terms of Schwinger parameters (the so-called parametric representation) the contribution to the amplitude coming from virtual quanta carrying arbitrarily high momenta is given by the contribution to the corresponding parametric integral coming from regions of the integration domain where all the Schwinger parameters are arbitrarily small. If we apply this reasoning to $i \Pi_{\mu \nu}^{\text {diam, }}(p)$, we conclude that, for $D \geqslant 4$, the non-integrable singularity in Eq. (2.10) at $t=0$ shows that virtual quanta $Q_{\mu}$ carrying arbitrarily high momenta yield an UV divergent contribution. This divergence needs renormalization. On the other hand, if $\tilde{p}^{2} \neq 0$, the integrand of any of the integrals in Eq. (2.11) is non-singular at $t=0$. Hence the 
contribution to $i \Pi_{\mu \nu}^{\text {diam, } \mathrm{NP}}(p)$ coming from arbitrary high momenta quanta $Q_{\mu}$ is curbed by the non-commutativity of space through the exponential $\exp \left(-\tilde{p}^{2} / 4 t\right)$, acting $1 / \tilde{p}^{2}$ as a regulator. Of course, if the regulator is removed, divergences spring back. This explains the UV origin of the IR divergences that occur in $i \Pi_{\mu \nu}^{\text {diam }, \mathrm{NP}}(p)$ at $\tilde{p}^{2}=0$. Furthermore, as it will come up shortly, the fact that the tree-level vertices vanish as $\theta^{\mu \nu} \rightarrow 0$, so that loops formally vanish in this limit, makes the coefficient of the logarithmic UV divergence in $i \Pi_{\mu \nu}^{\text {diam, }}$ P $(p)$ at $D=4$ to be opposite to the coefficient of its logarithmic IR divergence at $\tilde{p}^{2}=0$. All this is the UV/IR mixing at work [10].

The one-loop paramagnetic contribution $i \Pi_{\mu \nu}^{\text {param }}(p)$ to the vacuum polarization tensor is given by the contribution involving only two background fields which comes from the diagram in Fig. 4. For the diagram itself the Feynman rules yield

$$
\left\langle F_{\mu \nu}(-p) F_{\rho \sigma}(p)\right\rangle_{1 \mathrm{PI}}=4\left(\eta_{\mu \rho} \eta_{\nu \sigma}-\eta_{\mu \sigma} \eta_{\nu \rho}\right) J(p),
$$

where $J(p)$ is the integral

$$
J(p)=\int \frac{d^{D} q}{(2 \pi)^{D}} 2 \sin ^{2}\left(\frac{q \wedge p}{2}\right) \frac{1}{q^{2}(p+q)^{2}} .
$$

The contribution $i \Pi_{\mu \nu}^{\mathrm{param}}(p)$ is then given by

$$
i \Pi_{\mu \nu}^{\mathrm{param}}(p)=[8] i\left(p^{2} \eta_{\mu \nu}-p_{\mu} p_{\nu}\right) J(p),
$$

which has a planar and a non-planar part

$$
i \Pi_{\mu \nu}^{\mathrm{param}}(p)=i \Pi_{\mu \nu}^{\mathrm{param}, \mathrm{P}}(p)+i \Pi_{\mu \nu}^{\mathrm{param}, \mathrm{NP}}(p) .
$$

Using the same arguments as for the diamagnetic part, we obtain

$$
\begin{aligned}
i \Pi_{\mu \nu}^{\mathrm{param}, \mathrm{P}}(p) & =[8] i\left(p^{2} \eta_{\mu \nu}-p_{\mu} p_{\nu}\right) J^{\mathrm{P}}(p), \\
J^{\mathrm{P}}(p) & =\frac{1}{(4 \pi)^{D / 2}} \int_{0}^{1} d x \int_{0}^{\infty} d t t^{1-D / 2} e^{-p^{2} t x(1-x)}
\end{aligned}
$$

for the planar part, and

$$
\begin{aligned}
& i \Pi_{\mu \nu}^{\text {param,NP}}(p)=[8] i\left(p^{2} \eta_{\mu \nu}-p_{\mu} p_{\nu}\right) J^{\mathrm{NP}}(p), \\
& J^{\mathrm{NP}}(p)=-\frac{1}{(4 \pi)^{D / 2}} \int_{0}^{1} d x \int_{0}^{\infty} d t t^{1-D / 2} e^{-p^{2} t x(1-x)-\tilde{p}^{2} / 4 t}
\end{aligned}
$$

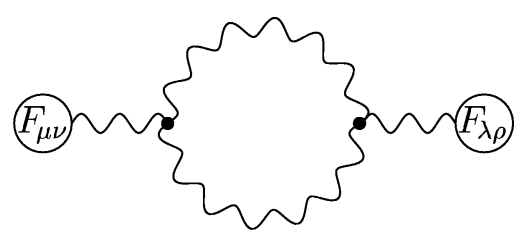

Fig. 4. One-loop paramagnetic diagram contributing to the 1PI Green function $\left\langle F_{\mu \nu}(-p) F_{\lambda \rho}(p)\right\rangle$. The diagram includes planar and non-planar terms. 
for the non-planar part. A similar analysis as for the diamagnetic contributions is in order. Indeed, the high momenta modes going around the loop of the paramagnetic diagram in Fig. 4 give, for $D \geqslant 4$, an UV divergent contribution to $J^{\mathrm{P}}(p)$, hence to $i \Pi_{\mu \nu}^{\mathrm{param}, \mathrm{P}}(p)$, which corresponds to the non-integrable singularity at $t=0$ in Eq. (2.15). The contribution of these modes to $J^{\mathrm{NP}}(p)$, hence to $i \Pi_{\mu \nu}^{\mathrm{NP}}(p)$ is finite provided $\tilde{p}^{2} \neq 0$. We may then say that the non-commutative character of space makes $1 / \tilde{p}^{2}$ to play the rôle of an UV regulator for the high-momentum modes contributing to the non-planar part of $i \Pi_{\mu \nu}^{\mathrm{param}}(p)$. If we remove the regulator, i.e., take the limit $1 / \tilde{p}^{2} \rightarrow \infty\left(\tilde{p}^{2} \rightarrow 0\right)$, the divergence is recovered, although this time under the guise of an IR divergence.

We are now ready to compute the contributions to the one-loop beta function of the theory at $D=4$ coming from the diamagnetic and paramagnetic parts of the vacuum polarization tensor. Recall that the mixed part vanishes. If we set $D=4+2 \varepsilon$ in Eqs. (2.10) and (2.15) and make a Laurent expansion around $\varepsilon=0$, we obtain

$$
\begin{gathered}
i \Pi_{\mu \nu}^{\text {diam }, \mathrm{P}}(p)=i\left(p^{2} \eta_{\mu \nu}-p_{\mu} p_{\nu}\right)\left[\frac{a^{\text {diam }}}{\varepsilon}+a^{\text {diam }} \ln \left(-\frac{p^{2}}{4 \pi}\right)-\frac{5}{72 \pi^{2}}+O(\varepsilon)\right], \\
i \Pi_{\mu \nu}^{\text {param }, \mathrm{P}}(p)=i\left(p^{2} \eta_{\mu \nu}-p_{\mu} p_{\nu}\right)\left[\frac{a^{\text {param }}}{\varepsilon}+a^{\text {param }} \ln \left(-\frac{p^{2}}{4 \pi}\right)+\frac{1}{\pi^{2}}+O(\varepsilon)\right],
\end{gathered}
$$

where

$$
a^{\text {diam }}=\frac{1}{16 \pi^{2}}\left[\frac{2}{3}\right], \quad a^{\text {param }}=\frac{1}{16 \pi^{2}}[-8] .
$$

Note that the coefficients $a^{\text {diam }}$ and $a^{\text {param }}$ can be obtained from the corresponding coefficients in Eq. (1.4) by replacing in the latter $N$ with 2. To subtract UV divergences, we work in the MS renormalization scheme and add to the classical action $S_{\text {class }}[B]$ the counterterm

$$
\delta S[B]=\frac{1}{4 \varepsilon}\left(a^{\text {diam }}+a^{\text {param }}\right) \int d^{D} x F^{\mu \nu}[B] \star F_{\mu \nu}[B],
$$

since its term quadratic in the background field $B_{\mu}$ cancels the UV divergences in Eqs. (2.17). In Sections 3 and 4 we will see that the UV divergences in the three and fourpoint function are also subtracted by the counterterm (2.19). Taking now into account that the tree-level part of the vacuum polarization tensor in terms of the bare coupling constant $g_{0}^{2}$ reads

$$
-\frac{i}{g_{0}^{2}}\left(p^{2} \eta_{\mu \nu}-p_{\mu} p_{\nu}\right)
$$

and using Eqs. (2.17), we conclude that up to one loop the renormalized vacuum polarization tensor in the MS scheme takes the form

$$
\begin{aligned}
i \Pi_{\mu \nu}^{\mathrm{ren}}(p)= & i\left(p^{2} \eta_{\mu \nu}-p_{\mu} p_{\nu}\right)\left[-\frac{1}{g^{2}}+\left(a^{\mathrm{diamag}}+a^{\mathrm{param}}\right) \ln \left(-\frac{p^{2}}{4 \pi \mu^{2}}\right)+\frac{67}{72 \pi^{2}}\right] \\
& +i \Pi_{\mu \nu}^{\mathrm{diam}, \mathrm{NP}}(p)+i \Pi_{\mu \nu}^{\mathrm{param}, \mathrm{NP}}(p),
\end{aligned}
$$


where the non-planar contributions $i \Pi_{\mu \nu}^{\mathrm{diam}, \mathrm{NP}}(p)$ and $i \Pi_{\mu \nu}^{\mathrm{param}, \mathrm{NP}}(p)$ are obtained by setting $D=4$ in Eqs. (2.11) and (2.16), respectively. The letter $g$ in Eq. (2.20) is the renormalized coupling constant in the MS scheme, given by Eq. (1.5) for $a^{\text {diam }}$ and $a^{\text {param }}$ as in Eq. (2.18). The beta function of the theory then reads

$$
\beta\left(g^{2}\right)=\mu \frac{d g^{2}}{d \mu}=2 g^{2}(\mu)\left(a^{\text {diam }}+a^{\text {param }}\right)=\frac{1}{4 \pi^{2}} g^{4}(\mu)\left[-\frac{11}{3}\right] .
$$

We see that the negative sign of the beta function comes about because the highmomentum paramagnetic contributions, which yield the coefficient $a^{\text {param }}$, overcome the high-momentum diamagnetic contributions, which originate the coefficient $a^{\text {diam }}$. The analogy with $S U(N)$ theory on commutative Minkowski spacetime is clear.

Let us next show that the dominance at high energies of paramagnetic contributions over diamagnetic ones has, due to the UV/IR mixing, a cut effect on the IR behaviour ${ }^{2}$ of the theory at $\tilde{p}=0$. We start by noting that the divergent IR behaviour at $\tilde{p}=0$ of the renormalized vacuum polarization tensor in Eq. (2.20) is only caused by $i \Pi_{\mu \nu}^{\text {diam, }} \mathrm{NP}(p)$ and $i \Pi_{\mu \nu}^{\text {param, } \mathrm{NP}}(p)$. The $\tilde{p}=0$ divergent terms of the latter are easily computed from Eqs. (2.11) and (2.16). For them we obtain

$$
\begin{aligned}
& i \Pi_{\mu \nu}^{\text {diam }, \mathrm{NP}}(p) \approx-i a^{\text {diam }} \ln \left(-p^{2} \tilde{p}^{2}\right)\left(p^{2} \eta_{\mu \nu}-p_{\mu} p_{\nu}\right)+\frac{2 i}{\pi^{2}} \frac{\tilde{p}_{\mu} \tilde{p}_{v}}{\left(\tilde{p}^{2}\right)^{2}}, \\
& i \Pi_{\mu \nu}^{\text {param }, \mathrm{NP}}(p) \approx-i a^{\text {param }} \ln \left(-p^{2} \tilde{p}^{2}\right)\left(p^{2} \eta_{\mu \nu}-p_{\mu} p_{\nu}\right),
\end{aligned}
$$

where $\approx$ means that we have dropped any term which is finite at $D=4$ as $\tilde{p} \rightarrow 0$. It is plain that the IR divergent logarithmic contributions in Eqs. (2.22) are dual to the UV divergent logarithmic terms in Eqs. (2.17), in the sense that in the momenta region

$$
|\tilde{p}| \sim \theta \Lambda_{\mathrm{IR}}
$$

the former can be obtained from the latter by using the replacements

$$
\frac{1}{\varepsilon} \leftrightarrow \ln \Lambda_{\mathrm{UV}}^{2}, \quad \Lambda_{\mathrm{UV}} \leftrightarrow \frac{1}{\theta \Lambda_{\mathrm{IR}}} .
$$

By contrast, the quadratic IR divergent term $\tilde{p}_{\mu} \tilde{p}_{v} /\left(\tilde{p}^{2}\right)^{2}$ in Eq. (2.22) has no dual counterpart as a singular UV contribution in Eq. (2.17). This is due to the fact that, if gauge invariance is preserved, no local and quadratic UV divergent contribution occurs in the vacuum polarization tensor. Yet, as can be seen from Eq. (2.11), the origin of both logarithmic and quadratic divergences at $\tilde{p}=0$ is the same: the curbing by the noncommutative character of the space, through the exponential $\exp \left(-\tilde{p}^{2} / 4 t\right)$, of the nonplanar contribution coming from the high-momentum quanta flowing along the loop of the diagrams in Fig. 3. Note that both the diamagnetic and paramagnetic functionals $S^{\text {diam }}$ and $S^{\text {param }}$ in Eq. (2.4) contribute to the non-commutative IR logarithmic divergence of the

\footnotetext{
${ }^{2}$ The IR divergences that occur when certain linear combinations $l_{i}(p)$ of the external momenta $p_{j}$ satisfy $\tilde{l}_{i}=0$, with $l_{i} \neq 0$, will be called non-commutative IR divergences throughout this paper. The behaviour of the Green functions in the neibourhood of those momentum configurations will be called non-commutative IR behaviour.
} 
vacuum polarization tensor, whereas the quadratic divergence only receives contributions from the diamagnetic functional, describing the orbital motion of $D-2$ scalar quanta in the field $B_{\mu}$. We shall see in the next section that the non-logarithmic IR divergences at $\tilde{p}=0$ of the one-loop three-point function also have a purely diamagnetic origin. Finally, adding the contributions in Eq. (2.22), we conclude that

$$
\begin{aligned}
i \Pi_{\mu \nu}(p) & \approx \frac{i}{16 \pi^{2}}\left[\frac{22}{3}\right] \ln \left(-p^{2} \tilde{p}^{2}\right)\left(p^{2} \eta_{\mu \nu}-p_{\mu} p_{\nu}\right)+\frac{2 i}{\pi^{2}} \frac{\tilde{p}_{\mu} \tilde{p}_{\nu}}{\tilde{p}^{4}} \\
& \equiv i \Pi_{\mu \nu}^{\mathrm{IR}}(p) .
\end{aligned}
$$

We have thus shown that the phenomenon of paramagnetic dominance at high energies, $\left|a^{\text {param }}\right|>a^{\text {diam }}$, which explains the sign of the one-loop beta function, also renders, via UV/IR mixing, the coefficient of the logarithmic divergence $\ln \left(-p^{2} \tilde{p}^{2}\right)$ in the vacuum polarization tensor equal to $22 / 3$. This coefficient and the coefficient of $\ln \left(-p^{2} / 4 \pi \mu^{2}\right)$ in Eq. (2.20) have the same absolute value but opposite sign, yet another manifestation of the mixing.

We shall close this section by showing that the right-hand side of Eqs. (2.10), (2.11), (2.15) and (2.16) also arise in the infinite tension limit of open string theory in a constant magnetic field. We will follow Ref. [15] and obtain the non-commutative $U(1)$ theory as the infinite tension limit of an open bosonic string theory on a D3-brane stuck at an $\mathbb{R}^{22} / Z_{2}$ orbifold singularity with a constant magnetic field along the worldvolume directions of the brane. The reader is referred to Ref. [15] for a comprehensive discussion. Let us first compute the one-loop non-planar contribution to the string amplitude with two photon vertex insertions of polarizations $e_{\mu}^{(1)}$ and $e_{v}^{(2)}$. This is given by

$$
\mathcal{A}^{\mathrm{NP}}=e_{\mu}^{(1)} i \Pi^{\mathrm{NP} \mu \nu} e_{\nu}^{(2)},
$$

where

$$
\begin{aligned}
i \Pi^{\mathrm{NP} \mu \nu}= & g^{2} \int_{0}^{\infty} \frac{d t}{2 t} \int_{0}^{2 \pi t} d y_{1} \int_{0}^{2 \pi t} d y_{2} Z(t) \\
& \times\left(-p_{\rho} p_{\sigma} \partial_{y_{1}} \widetilde{\mathcal{G}}^{\rho \sigma} \partial_{y_{2}} \widetilde{\mathcal{G}}^{\mu \nu}+p_{\rho} p_{\sigma} \partial_{y_{1}} \widetilde{\mathcal{G}}^{\mu \rho} \partial_{y_{2}} \widetilde{\mathcal{G}}^{v \sigma}\right) e^{p_{\rho} p_{\sigma}} \widetilde{\mathcal{G}}^{\rho \sigma},
\end{aligned}
$$

$Z(t)$ is the open string partition function of an open bosonic string ending on a $\mathrm{D} p$-brane glued at an orbifold singularity in the presence of a constant magnetic field along the directions of the brane, and $\widetilde{\mathcal{G}}^{\mu \nu}$ is related to the string propagator and reads

$$
\widetilde{\mathcal{G}}^{\mu \nu}=-\alpha^{\prime}\left[G^{\mu v} \Gamma^{\mathrm{NP}}(y \mid t)+\frac{i \theta^{\mu \nu}}{2 \pi \alpha^{\prime}} \frac{y}{t}+\frac{(\theta G \theta)^{\mu \nu}}{\left(2 \pi \alpha^{\prime}\right)^{2}} \frac{\pi}{2 t}\right] .
$$

Here $G^{\mu \nu}$ is the inverse of the effective open string metric $G_{\mu \nu}$ [19], $y=y_{1}-y_{2}$ and $\Gamma^{\mathrm{NP}}$ has the form

$$
\Gamma^{\mathrm{NP}}(y \mid t)=\ln \left|2 \pi \frac{\vartheta_{2}(i y / 2 \pi, i t)}{\vartheta_{1}^{\prime}(0, i t)}\right|^{2}-\frac{y^{2}}{2 \pi t} .
$$


From Eqs. (2.27) and (2.26), one readily has

$$
\begin{aligned}
i \Pi^{\mathrm{NP} \mu \nu}= & g^{2} \int_{0}^{\infty} \frac{d t}{2 t} \int_{0}^{2 \pi t} d y_{1} \int_{0}^{2 \pi t} d y_{2} Z(t) \\
& \times \alpha^{\prime 2}\left\{\left(p^{2} G^{\mu \nu}-p^{\mu} p^{v}\right)\left[\partial_{y} \Gamma^{\mathrm{NP}}(y \mid t)\right]^{2}\right. \\
& \left.\quad+\frac{i\left(p^{2} \theta^{\mu \nu}-p^{\mu} \tilde{p}^{v}-p^{v} \tilde{p}^{\mu}\right)}{2 \pi \alpha^{\prime}} \frac{1}{t} \partial_{y} \Gamma^{\mathrm{NP}}(y \mid t)+\frac{\tilde{p}^{\mu} \tilde{p}^{v}}{\left(2 \pi \alpha^{\prime}\right)^{2}} \frac{1}{t^{2}}\right\} \\
& \times e^{-\alpha^{\prime} p^{2} \Gamma^{\mathrm{NP}}(y \mid t)} e^{-\tilde{p}^{2} / 8 \pi \alpha^{\prime} t},
\end{aligned}
$$

with $p^{2}=G_{\mu \nu} p^{\mu} p^{v}, p_{v}=G_{v \mu} p^{\mu}$ and $\tilde{p}^{\mu}=\theta^{\mu v} p_{v}$. To obtain from this expression the corresponding field theory result, one needs the large $t$ expansion of the functions in the integrand upon performing the change of variables $y=2 \pi x t$. The relevant terms of these expansions are

$$
\begin{aligned}
Z(t) & \sim \frac{V_{d}}{\left(8 \pi^{2} \alpha^{\prime} t\right)^{d / 2}}\left(e^{2 \pi t}+d-2\right), \\
\Gamma^{\mathrm{NP}}(2 \pi x t \mid t) & \sim 2 \pi t x(1-x), \\
\left.\partial_{y} \Gamma^{\mathrm{NP}}(y \mid t)\right|_{y=2 \pi x t} & \sim 1-2 x+2\left(e^{-2 \pi t} e^{2 \pi x t}-e^{-2 \pi x t}\right),
\end{aligned}
$$

where we have taken a $\mathrm{D}(d-1)$-brane to compute $Z(t)$. Substituting these expansions in Eq. (2.29) and making the changes of scale $t \rightarrow t / \alpha^{\prime}$ and $y \rightarrow y / \alpha^{\prime}$, one obtains after some calculations ${ }^{3}$ the non-planar contribution to the vacuum polarization tensor as the sum

$$
i \Pi_{\mu \nu}^{\mathrm{NP}}(p)=i \Pi_{\mu \nu}^{\mathrm{NP}, \operatorname{tach}}(p)+i \Pi_{\mu \nu}^{\mathrm{NP}, 0-\operatorname{mode}}(p)
$$

of two terms:

$$
\begin{aligned}
& i \Pi_{\mu \nu}^{\mathrm{NP}, 0-\operatorname{mode}}(p) \\
& =i g_{0}^{2} \frac{[d-2]}{(4 \pi)^{d / 2}}\left[\left(p^{2} G_{\mu \nu}-p_{\mu} p_{\nu}\right) \int_{0}^{1} d x(1-2 x)^{2} \int_{0}^{\infty} d t t^{1-d / 2} e^{-p^{2} t x(1-x)-\tilde{p}^{2} / 4 t}\right. \\
& \left.+\tilde{p}_{\mu} \tilde{p}_{v} \int_{0}^{1} d x \int_{0}^{\infty} d t t^{-1-d / 2} e^{-p^{2} t x(1-x)-\tilde{p}^{2} / 4 t}\right]
\end{aligned}
$$

and

$$
\begin{aligned}
i & \Pi_{\mu \nu}^{\mathrm{NP}, \operatorname{tach}}(p) \\
& =-i g_{0}^{2} \frac{[-8]}{(4 \pi)^{d / 2}}\left(p^{2} G_{\mu \nu}-p_{\mu} p_{\nu}\right) \int_{0}^{1} d x \int_{0}^{\infty} d t t^{1-d / 2} e^{-p^{2} t x(1-x)-\tilde{p}^{2} / 4 t} .
\end{aligned}
$$

The same arguments as those used in Ref. [5] (see also Ref. [6]) for Yang-Mills theory on commutative Minkowski spacetime show that $i \Pi_{\mu \nu}^{\mathrm{NP}, 0 \text {-mode }}(p)$ originates from the

\footnotetext{
${ }^{3}$ This includes dropping divergent contributions as $t \rightarrow \infty$ for arbitrary $x$.
} 
product of the one-loop photon contribution to the partition function and the zero-mode contribution to the string Green function. In turn, $i \Pi_{\mu \nu}^{\mathrm{NP}}$, tach $(p)$ arises from the combination of the tachyonic contribution to the partition function and the exponentially vanishing part of the string Green function, an effect called tachyon magnification in Ref. [5]. This can be understood as follows.

Indeed, the contribution proportional to $p^{2} G_{\mu \nu}-p_{\mu} p_{v}$ in $i \Pi_{\mu \nu}^{\mathrm{NP}, 0-\text { mode }}(p)$ arises from combining the term $d-2$ in Eq. (2.30), carrying the one-loop photon contribution to $Z(t)$, with the square of the zero-mode term $1-2 x$ in Eq. (2.32). And the term proportional to $\tilde{p}_{\mu} \tilde{p}_{\nu}$ in $i \Pi_{\mu \nu}^{\mathrm{NP}, 0 \text {-mode }}(p)$, note that it comes with the extra zero-mode factor $1 / t^{2}$. As concerns $i \Pi_{\mu \nu}^{\mathrm{NP}}$, tach $(p)$, we note that it originates from the term in Eq. (2.29) proportional to $p^{2} G_{\mu \nu}-p_{\mu} p_{v}$ that goes with the product

$$
e^{2 \pi t} \times 4\left(e^{-2 \pi t} e^{2 \pi x t}-e^{-2 \pi x t}\right)^{2} .
$$

The first factor in this product, $e^{2 \pi t}$, is supplied by the large- $t$ expansion of $Z(t)$ and is due to the open string tachyon. The second factor arises from squaring the term $2\left(e^{-2 \pi t} e^{2 \pi x t}-\right.$ $\left.e^{-2 \pi x t}\right)$ in Eq. (2.32) and has its origin in the open string propagator. It is precisely $-8 e^{2 \pi t}$, the evanescent large- $t$ and $x$-independent contribution to this second factor, which is magnified when combined with the $t$-divergent tachyon contribution to $Z(t)$, all in all yielding a finite non-zero result. Note that Eqs. (2.33) and (2.34) agree with Eqs. (2.11) and (2.16), if we identify $G_{\mu \nu}$ with $\eta_{\mu \nu}$ and $d$ with $D$. Hence we have identified the diamagnetic and paramagnetic non-planar contributions in field theory with the zero-mode and tachyon magnification contributions in string theory, an observation first made for Yang-Mills theory in commutative Minkowski spacetime in Ref. [5].

A similar analysis can be performed for the planar contribution to the string amplitude with two photon vertex insertions. It is this contribution that develops an UV divergence when the field theory limit is taken. Furthermore, in this limit, this contribution can be decomposed in the sum of a zero-mode part and a tachyon magnification part, whose expressions agree with the r.h.s. of Eqs. (2.10) and (2.15), respectively. Thus, the charge screening contribution to the beta function - given by the coefficient $a_{1}^{\text {diam }}$ - is concocted by the diamagnetic contributions in the field theory setting and has a zero mode origin in string theory framework. On the other hand, the charge anti-screening contribution provided by $a_{1}^{\text {param }}$ — has a paramagnetic origin in field theory and is the result of tachyon magnification in string theory.

\section{The three-point function}

In this section we study, in the light of high-energy paramagnetic dominance, the UV and non-commutative IR divergences of the three-point function and their mixing. The 1PI three-point function $\Gamma_{\mu_{1} \mu_{2} \mu_{3}}\left(x_{1}, x_{2}, x_{3}\right)$ is defined as

$$
i \Gamma_{\mu_{1} \mu_{2} \mu_{3}}\left(x_{1}, x_{2}, x_{3}\right)=\left.\frac{\delta^{3} \Gamma[B]}{\delta B_{\mu_{1}}\left(x_{1}\right) \delta B_{\mu_{2}}\left(x_{2}\right) \delta B_{\mu_{3}}\left(x_{3}\right)}\right|_{B=0},
$$


where $i \Gamma[B]=\ln Z[B]$ and $Z[B]$ is given in Eq. (2.3). In momentum space we will write $i \Gamma_{\mu_{1} \mu_{2} \mu_{3}}\left(p_{1}, p_{2}, p_{3}\right)$, with $p_{1}+p_{2}+p_{3}=0$. The three-point function can be expressed as the sum of a diamagnetic contribution, which sums over diagrams with all vertices of diamagnetic type, a paramagnetic contribution, given by diagrams with only paramagnetic vertices, and a mixed contribution, made of diagrams with at least one vertex of each type:

$$
\begin{aligned}
i \Gamma_{\mu_{1} \mu_{2} \mu_{3}}\left(p_{1}, p_{2}, p_{3}\right)= & i \Gamma_{\mu_{1} \mu_{2} \mu_{3}}^{\mathrm{diam}}\left(p_{1}, p_{2}, p_{3}\right)+i \Gamma_{\mu_{1} \mu_{2} \mu_{3}}^{\mathrm{param}}\left(p_{1}, p_{2}, p_{3}\right) \\
& +i \Gamma_{\mu_{1} \mu_{2} \mu_{3}}^{\text {mixed }}\left(p_{1}, p_{2}, p_{3}\right) .
\end{aligned}
$$

We start computing the diamagnetic contribution $i \Gamma_{\mu_{1} \mu_{2} \mu_{3}}^{\mathrm{diam}}\left(p_{1}, p_{2}, p_{3}\right)$ to the three-point function. The one-loop diamagnetic Feynman diagrams that contribute to this function are shown in Fig. 5. The Feynman diagrams in the first row will be called triangle diagrams, while the remaining diagrams will be referred to as swordfish diagrams. The sum $i \Gamma_{\mu_{1} \mu_{2} \mu_{3}}^{\text {triang }}\left(p_{1}, p_{2}, p_{3}\right)$ of the triangle diagrams is formally given in $D$ dimensions by the integral

$$
\begin{aligned}
(\mathrm{a})+ & (\mathrm{b})+(\mathrm{c}) \\
= & i \Gamma_{\mu_{1} \mu_{2} \mu_{3}}^{\text {triang }}\left(p_{1}, p_{2}, p_{3}\right) \\
= & 8 i[D-2] \int \frac{d^{D} q}{(2 \pi)^{D}} \frac{\left(2 q+p_{1}\right)_{\mu_{1}}\left(2 q+2 p_{1}+p_{2}\right)_{\mu_{2}}\left(2 q+p_{1}+p_{2}\right)_{\mu_{3}}}{q^{2}\left(q+p_{1}\right)^{2}\left(q+p_{1}+p_{2}\right)^{2}} \\
& \times \sin \left[\frac{q \wedge p_{1}}{2}\right] \sin \left[\frac{\left(q+p_{1}\right) \wedge p_{2}}{2}\right] \sin \left[\frac{q \wedge\left(p_{1}+p_{2}\right)}{2}\right] .
\end{aligned}
$$

The sum $i \mathrm{I}_{\mu_{1} \mu_{2} \mu_{3}}^{\text {sfish }}\left(p_{1}, p_{2}, p_{3}\right)$ of the swordfish diagrams (d) and (g), also in $D$ dimensions, is given by

$$
\begin{aligned}
(\mathrm{d})+ & (\mathrm{g}) \\
= & i \mathrm{I}_{\mu_{1} \mu_{2} \mu_{3}}^{\mathrm{sfish}}\left(p_{1}, p_{2}, p_{3}\right) \\
= & 4 i[D-2] \eta_{\mu_{1} \mu_{2}} \int \frac{d^{D} q}{(2 \pi)^{D}} \frac{\left(2 q+p_{1}+p_{2}\right)_{\mu_{3}}}{q^{2}\left(q+p_{1}\right)^{2}\left(q+p_{1}+p_{2}\right)^{2}} \sin \left[\frac{q \wedge\left(p_{1}+p_{2}\right)}{2}\right] \\
& \times\left\{\sin \left[\frac{\left(q+p_{1}\right) \wedge p_{2}}{2}\right] \sin \left[\frac{q \wedge p_{1}}{2}\right]+\sin \left[\frac{\left(q+p_{2}\right) \wedge p_{1}}{2}\right] \sin \left[\frac{q \wedge p_{2}}{2}\right]\right\},
\end{aligned}
$$

and the total swordfish contribution $i \Gamma_{\mu_{1} \mu_{2} \mu_{3}}^{\mathrm{sfish}}\left(p_{1}, p_{2}, p_{3}\right)$ reads

$$
\begin{aligned}
i & \Gamma_{\mu_{1} \mu_{2} \mu_{3}}^{\mathrm{sfish}}\left(p_{1}, p_{2}, p_{3}\right) \\
& =(\mathrm{d})+(\mathrm{g})+(\mathrm{e})+(\mathrm{h})+(\mathrm{f})+(\mathrm{i}) \\
& =i \mathrm{I}_{\mu_{1} \mu_{2} \mu_{3}}^{\mathrm{sfish}}\left(p_{1}, p_{2}, p_{3}\right)+i \mathrm{I}_{\mu_{3} \mu_{2} \mu_{1}}^{\mathrm{sfish}}\left(p_{3}, p_{2}, p_{1}\right)+i \mathrm{I}_{\mu_{1} \mu_{3} \mu_{2}}^{\mathrm{sfish}}\left(p_{1}, p_{3}, p_{2}\right) .
\end{aligned}
$$

As in the vacuum polarization tensor case, the overall factors $[D-2]$ in Eqs. (3.1) and (3.2) account for the fact that the one-loop diamagnetic contribution is due to the orbital motion of $D-2$ real scalar quanta in the background field $B_{\mu}$. The $D$ comes from the diagrams with fields $Q_{\mu}(\mu=1, \ldots, D)$ going around the loop, while the -2 is provided by the corresponding diagrams with ghost loops. Each diagram in Fig. 5 


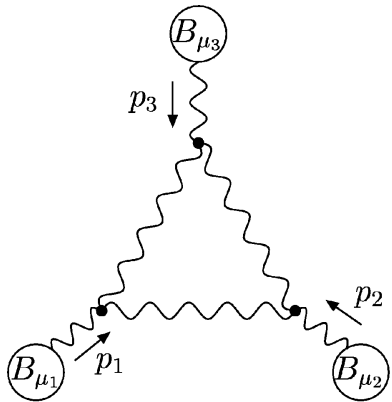

(a)

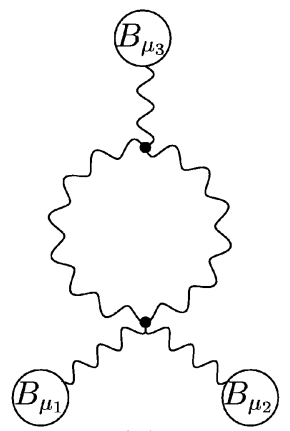

(d)

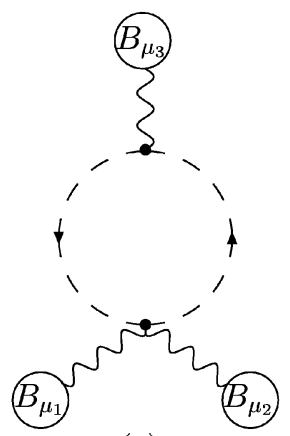

(g)

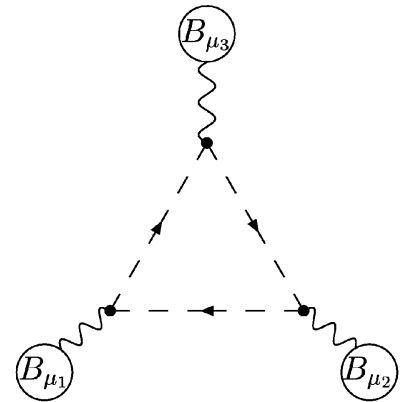

(b)

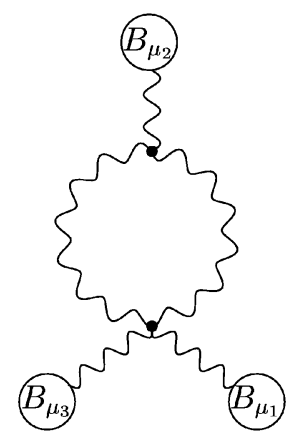

(e)

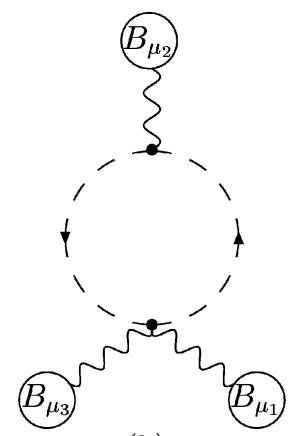

(h)

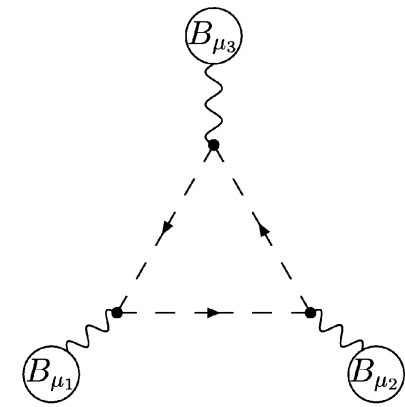

(c)

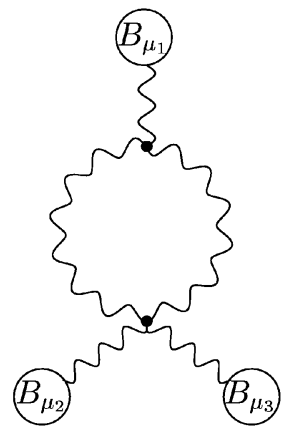

(f)

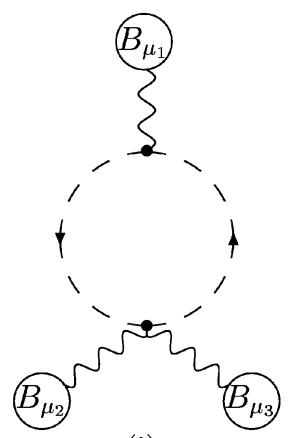

(i)

Fig. 5. One-loop 1PI diamagnetic diagrams contributing to the three-point function.

can be expressed as the sum of its planar part and its non-planar part by repeated use of the identity $2 i \sin \left(\frac{1}{2} q \wedge p\right)=e^{i q \wedge p / 2}-e^{-i q \wedge p / 2}$. The planar part is the contribution whose $\theta^{\mu \nu}$-dependent complex exponential factors do not depend on the loop momenta, while the non-planar part collects all terms whose $\theta^{\mu \nu}$-dependent complex exponentials depend on the loop momenta. At $D=4$, the planar parts of the diagrams in Fig. 5 are UV divergent by power counting. This UV divergence is logarithmic and shows in dimensional 
regularization as a simple pole at $D=4$. By contrast, the non-planar parts are finite since the UV divergence is tamed by the the exponentials $e^{i q \wedge p_{i}}$, provided $p_{i} \neq 0$. In other words, the contribution to the integrals in Eqs. (3.1) and (3.2) of the quantum modes flowing along the loop with arbitrarily high momenta is UV divergent when one considers their planar part, and it is turned into an IR divergence by the non-commutativity of space when one looks at their non-planar parts. This IR divergence occurs whenever any of the conditions

$$
\tilde{p}_{1}=0, \quad \tilde{p}_{2}=0, \quad \tilde{p}_{3}=0
$$

is met. This provides a qualitative explanation of the UV/IR for the diamagnetic part of the three-point function. To make it quantitative, we need to compute the UV divergent terms in the planar contribution and the non-commutative IR divergent parts of the non-planar contribution.

To calculate the UV divergence of the planar contribution, we make a Laurent expansion about $D=4$ of the corresponding dimensionally regularized integrals and retain singular contributions. Proceeding in this way and defining $D=4+2 \varepsilon$, we obtain for nonexceptional external momenta

$$
\begin{aligned}
& i \Gamma_{\mu_{1} \mu_{2} \mu_{3}}^{\operatorname{trian}, \mathrm{P}}\left(p_{1}, p_{2}, p_{3}\right) \\
& =\frac{-i}{16 \pi^{2} \varepsilon} \frac{2}{3} 2 i \sin \left(\frac{p_{1} \wedge p_{2}}{2}\right) \\
& \quad \times\left[\eta_{\mu_{1} \mu_{2}}\left(p_{2}-p_{1}\right)_{\mu_{3}}+\eta_{\mu_{1} \mu_{3}}\left(p_{1}-p_{3}\right)_{\mu_{2}}+\eta_{\mu_{2} \mu_{3}}\left(p_{3}-p_{2}\right)_{\mu_{1}}\right]+O\left(\varepsilon^{0}\right),
\end{aligned}
$$

for the sum of the triangle diagrams, and

$$
i \Gamma_{\mu_{1} \mu_{2} \mu_{3}}^{\mathrm{sfish}, \mathrm{P}}\left(p_{1}, p_{2}, p_{3}\right)=O\left(\varepsilon^{0}\right)
$$

for the sum of the swordfish diagrams. From this we conclude that the UV divergent contribution $i \Gamma_{\mu_{1} \mu_{2} \mu_{3}}^{\mathrm{diam}, \mathrm{UV}}\left(p_{1}, p_{2}, p_{3}\right)$ of the diamagnetic part to the three-point function is given in dimensional regularization by

$$
\begin{aligned}
& i \Gamma_{\mu_{1} \mu_{2} \mu_{3}}^{\mathrm{diam}, \mathrm{UV}}\left(p_{1}, p_{2}, p_{3}\right) \\
& =\frac{-i a_{1}^{\mathrm{diam}}}{\varepsilon} 2 i \sin \left(\frac{p_{1} \wedge p_{2}}{2}\right) \\
& \quad \times\left[\eta_{\mu_{1} \mu_{2}}\left(p_{2}-p_{1}\right)_{\mu_{3}}+\eta_{\mu_{1} \mu_{3}}\left(p_{1}-p_{3}\right)_{\mu_{2}}+\eta_{\mu_{2} \mu_{3}}\left(p_{3}-p_{2}\right)_{\mu_{1}}\right],
\end{aligned}
$$

where $a_{1}^{\text {diam }}$ is as in Eq. (2.18). To compute the IR divergences that appear in the non-planar contribution when one or more of the $\tilde{p}_{i}$ vanish, we set $D=4$ and use the following basic results for $\tilde{k} \rightarrow 0$ :

$$
\begin{aligned}
& \int \frac{d^{4} q}{(2 \pi)^{4}} \frac{e^{i q \wedge k}}{q^{2}\left(q+\ell_{1}\right)^{2}} \approx \frac{-i}{16 \pi^{2}} \ln \tilde{k}^{2} \\
& \int \frac{d^{4} q}{(2 \pi)^{4}} \frac{q_{\mu_{1}} q_{\mu_{2}} e^{i q \wedge k}}{q^{2}\left(q+\ell_{1}\right)^{2}\left(q+\ell_{2}\right)^{2}} \approx \frac{-i}{16 \pi^{2}} \frac{1}{4} \ln \tilde{k}^{2} \eta_{\mu_{1} \mu_{2}},
\end{aligned}
$$




$$
\begin{gathered}
\int \frac{d^{4} q}{(2 \pi)^{4}} \frac{q_{\mu_{1}} q_{\mu_{2}} q_{\mu_{3}} e^{i q \wedge k}}{q^{2}\left(q+\ell_{1}\right)^{2}\left(q+\ell_{2}\right)^{2}} \\
\approx \frac{i}{16 \pi^{2}}\left\{\frac{i}{2 \tilde{k}^{2}}\left[\eta_{\mu_{1} \mu_{2}} \tilde{k}_{\mu_{3}}+\eta_{\mu_{2} \mu_{3}} \tilde{k}_{\mu_{1}}+\eta_{\mu_{1} \mu_{3}} \tilde{k}_{\mu_{2}}-2 \frac{\tilde{k}_{\mu_{1}} \tilde{k}_{\mu_{2}} \tilde{k}_{\mu_{3}}}{\tilde{k}^{2}}\right]\right. \\
\quad+\frac{1}{12} \ln \tilde{k}^{2}\left[\eta_{\mu_{1} \mu_{2}}\left(\ell_{1}+\ell_{2}\right)_{\mu_{3}}+\eta_{\mu_{2} \mu_{3}}\left(\ell_{1}+\ell_{2}\right)_{\mu_{1}}\right. \\
\left.\left.+\eta_{\mu_{1} \mu_{3}}\left(\ell_{1}+\ell_{2}\right)_{\mu_{2}}\right]\right\},
\end{gathered}
$$

where $\ell_{1}$ and $\ell_{2}$ never vanish and $\approx$ indicates that we have dropped any contribution which remains finite as $\tilde{k} \rightarrow 0$. These expressions, together with some algebra, lead to the following divergences for the non-planar parts of the diagrams in Fig. 5 when one or more $\tilde{p}_{i}$ approach zero:

$$
\begin{aligned}
& i \Gamma_{\mu_{1} \mu_{2} \mu_{3}}^{\mathrm{triang}, \mathrm{NP}}\left(p_{1}, p_{2}, p_{3}\right) \\
& \approx \frac{i}{16 \pi^{2}} \frac{2}{9} 2 i \sin \left(\frac{p_{1} \wedge p_{2}}{2}\right)\left(\ln \tilde{p}_{1}^{2}+\ln \tilde{p}_{2}^{2}+\ln \tilde{p}_{3}^{2}\right) \\
& \quad \times\left[\eta_{\mu_{1} \mu_{2}}\left(p_{2}-p_{1}\right)_{\mu_{3}}+\eta_{\mu_{1} \mu_{3}}\left(p_{1}-p_{3}\right)_{\mu_{2}}+\eta_{\mu_{2} \mu_{3}}\left(p_{3}-p_{2}\right)_{\mu_{1}}\right] \\
& +\frac{1}{\pi^{2}} \cos \left(\frac{p_{1} \wedge p_{2}}{2}\right) \sum_{i=1}^{3}\left[\frac{2\left(\tilde{p}_{i}\right)_{\mu_{1}}\left(\tilde{p}_{i}\right)_{\mu_{2}}\left(\tilde{p}_{i}\right)_{\mu_{3}}}{\left(\tilde{p}_{i}^{2}\right)^{2}}-\eta_{\mu_{1} \mu_{2}} \frac{\left(\tilde{p}_{i}\right)_{\mu_{3}}}{\tilde{p}_{i}^{2}}\right. \\
& \left.\quad-\eta_{\mu_{2} \mu_{3}} \frac{\left(\tilde{p}_{i}\right)_{\mu_{1}}}{\tilde{p}_{i}^{2}}-\eta_{\mu_{1} \mu_{3}} \frac{\left(\tilde{p}_{i}\right)_{\mu_{2}}}{\tilde{p}_{i}^{2}}\right]
\end{aligned}
$$

and

$$
\begin{aligned}
& i \Gamma_{\mu_{1} \mu_{2} \mu_{3}}^{\mathrm{sfish}}\left(p_{1}, p_{2}, p_{3}\right) \\
& \quad \approx \frac{1}{\pi^{2}} \cos \left(\frac{p_{1} \wedge p_{2}}{2}\right) \sum_{i=1}^{3}\left[\eta_{\mu_{1} \mu_{2}} \frac{\left(\tilde{p}_{i}\right)_{\mu_{3}}}{\tilde{p}_{i}^{2}}+\eta_{\mu_{2} \mu_{3}} \frac{\left(\tilde{p}_{i}\right)_{\mu_{1}}}{\tilde{p}_{i}^{2}}+\eta_{\mu_{1} \mu_{3}} \frac{\left(\tilde{p}_{i}\right)_{\mu_{2}}}{\tilde{p}_{i}^{2}}\right] .
\end{aligned}
$$

Adding these two expressions we obtain for the non-commutative IR divergent part of the diamagnetic contribution to the three-point function

$$
\begin{aligned}
& i \Gamma_{\mu_{1} \mu_{2} \mu_{3}}^{\text {diam,IR }}\left(p_{1}, p_{2}, p_{3}\right) \\
& =\frac{2}{\pi^{2}} \cos \left(\frac{p_{1} \wedge p_{2}}{2}\right) \sum_{i=1}^{3} \frac{\left(\tilde{p}_{i}\right)_{\mu_{1}}\left(\tilde{p}_{i}\right)_{\mu_{2}}\left(\tilde{p}_{i}\right)_{\mu_{3}}}{\left(\tilde{p}_{i}^{2}\right)^{2}} \\
& \quad+\frac{i}{3} a^{\operatorname{diam}} 2 i \sin \left(\frac{p_{1} \wedge p_{2}}{2}\right)\left(\ln \tilde{p}_{1}^{2}+\ln \tilde{p}_{2}^{2}+\ln \tilde{p}_{3}^{2}\right) \\
& \quad \times\left[\eta_{\mu_{1} \mu_{2}}\left(p_{2}-p_{1}\right)_{\mu_{3}}+\eta_{\mu_{1} \mu_{3}}\left(p_{1}-p_{3}\right)_{\mu_{2}}+\eta_{\mu_{2} \mu_{3}}\left(p_{3}-p_{2}\right)_{\mu_{1}}\right],
\end{aligned}
$$

where $a_{1}^{\text {diam }}$ is given in Eq. (2.18). Notice that the logarithmic term in this expression is dual to the the UV divergent contribution given in Eq. (3.4), in the sense that in the momenta region

$$
\left|\tilde{p}_{1}\right| \sim\left|\tilde{p}_{2}\right| \sim\left|\tilde{p}_{3}\right| \sim \theta \Lambda_{\mathrm{IR}} \rightarrow 0
$$


the former can be obtained from the latter by using the identifications (2.24). Note also that the factor $\sin \left(\frac{1}{2}\left(p_{1} \wedge p_{2}\right)\right.$, together with momentum conservation $\tilde{p}_{1}+\tilde{p}_{2}+\tilde{p}_{3}=0$, renders the second term in Eq. (3.8) finite when $\tilde{p}_{i} \rightarrow 0(i=1,2,3)$. This is so in spite of the logarithmic non-commutative IR divergences of the integrals (3.5)-(3.7) that enter the computation of the non-planar part of the diamagnetic contribution, and can be thought of as a consequence of gauge invariance - which determines the momentum structure of the UV divergent part of the three-point function in Eq. (3.4) - and the UV/IR mixing mechanism. Indeed, UV/IR mixing in the form of Eq. (2.24) allows to retrieve from the UV divergent contribution $i \Gamma_{\mu_{1} \mu_{2} \mu_{3}}^{\text {diam, UV }}\left(p_{1}, p_{2}, p_{3}\right)$ the terms in $i \Gamma_{\mu_{1} \mu_{2} \mu_{3}}^{\text {diam, NP }}\left(p_{1}, p_{2}, p_{3}\right)$ which involve $\ln \tilde{p}_{i}^{2}$, with $\tilde{p}_{i}$ verifying Eq. (3.9).

Let us compute now the paramagnetic part $i \Gamma_{\mu_{1} \mu_{2} \mu_{3}}^{\mathrm{param}}\left(p_{1}, p_{2}, p_{3}\right)$ of the the three-point function. This is given by the contribution involving only three background fields that comes from the diagram in Fig. 4, which in turn is given by Eqs. (2.12) and (2.13). Extracting from Eq. (2.12) the contribution cubic in the background fields and using the results of section 2 for the integral $J(p)$, we obtain, after some calculations,

$$
\begin{aligned}
& i \Gamma_{\mu_{1} \mu_{2} \mu_{3}}^{\mathrm{param}}\left(p_{1}, p_{2}, p_{3}\right) \\
& =\frac{-i a^{\mathrm{param}}}{\varepsilon} 2 i \sin \left(\frac{p_{1} \wedge p_{2}}{2}\right) \\
& \quad \times\left[\eta_{\mu_{1} \mu_{2}}\left(p_{2}-p_{1}\right)_{\mu_{3}}+\eta_{\mu_{1} \mu_{3}}\left(p_{1}-p_{3}\right)_{\mu_{2}}+\eta_{\mu_{2} \mu_{3}}\left(p_{3}-p_{2}\right)_{\mu_{1}}\right]
\end{aligned}
$$

for the UV divergent part, and

$$
\begin{aligned}
i & \Gamma_{\mu_{1} \mu_{2} \mu_{3}}^{\mathrm{param}}\left(p_{1}, p_{2}, p_{3}\right) \\
= & \frac{i}{3} a^{\mathrm{param}} 2 i \sin \left(\frac{p_{1} \wedge p_{2}}{2}\right)\left(\ln \tilde{p}_{1}^{2}+\ln \tilde{p}_{2}^{2}+\ln \tilde{p}_{3}^{2}\right) \\
& \times\left[\eta_{\mu_{1} \mu_{2}}\left(p_{2}-p_{1}\right)_{\mu_{3}}+\eta_{\mu_{1} \mu_{3}}\left(p_{1}-p_{3}\right)_{\mu_{2}}+\eta_{\mu_{2} \mu_{3}}\left(p_{3}-p_{2}\right)_{\mu_{1}}\right]
\end{aligned}
$$

for the IR divergences that appear as $\tilde{p}_{i} \rightarrow 0$. Here $a_{1}^{\text {param }}$ is defined in Eq. (2.18). As in the case of the diamagnetic contribution, the IR part $i \Gamma_{\mu_{1} \mu_{2} \mu_{3}}^{\mathrm{param}, \mathrm{IR}}\left(p_{1}, p_{2}, p_{3}\right)$ is dual to the UV part $i \Gamma_{\mu_{1} \mu_{2} \mu_{3}}^{\mathrm{param}, \mathrm{UV}}\left(p_{1}, p_{2}, p_{3}\right)$, in the sense that one can go from one to another by using the identifications (2.24) in the momentum region (3.9).

We finally look at the mixed part $i \Gamma_{\mu_{1} \mu_{2} \mu_{3}}^{\text {mixed }}\left(p_{1}, p_{2}, p_{3}\right)$. It receives contributions from the diagrams in Figs. 2 and 6. As explained in Section 2, the diagram of Fig. 2 vanishes. The very same arguments as for the diagram in Fig. 2 show that the diagram in Fig. 6 (a) also vanishes. By contrast, the diagrams in Figs. 6 (b) and (c) are different from zero; yet their planar parts are finite by power counting and their non-planar parts are IR finite for vanishing $\tilde{p}_{i}$. Altogether, we conclude

$$
\Gamma_{\mu_{1} \mu_{2} \mu_{3}}^{\text {mixed,UV }}\left(p_{1}, p_{2}, p_{3}\right)=\Gamma_{\mu_{1} \mu_{2} \mu_{3}}^{\text {mixed,IR }}\left(p_{1}, p_{2}, p_{3}\right)=0 .
$$

The UV divergent part $i \Gamma_{\mu_{1} \mu_{2} \mu_{3}}^{\mathrm{UV}}\left(p_{1}, p_{2}, p_{3}\right)$ of the three-point function will be the sum of the diamagnetic, paramagnetic and mixed UV divergent parts. Using the results 


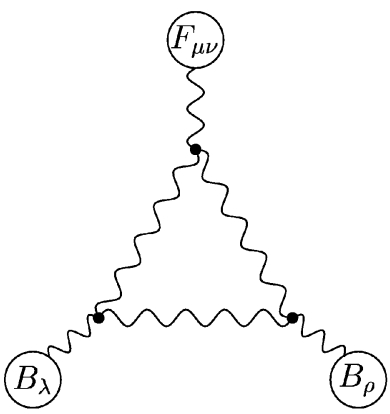

(a)

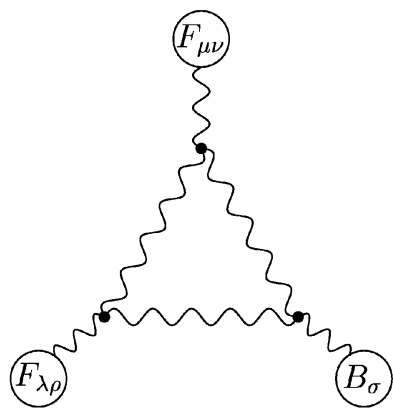

(b)

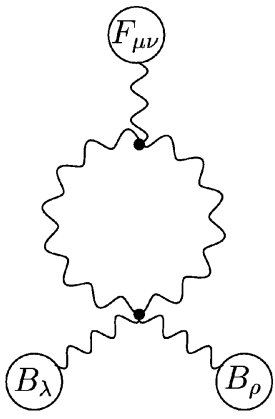

(c)

Fig. 6. One-loop mixed diagrams contributing to the three-point function.

obtained, we have

$$
\begin{aligned}
i & \Gamma_{\mu_{1} \mu_{2} \mu_{3}}^{\mathrm{UV}}\left(p_{1}, p_{2}, p_{3}\right) \\
= & \frac{-i}{\varepsilon}\left(a^{\mathrm{diam}}+a^{\mathrm{param}}\right) 2 i \sin \left(\frac{p_{1} \wedge p_{2}}{2}\right) \\
& \times\left[\eta_{\mu_{1} \mu_{2}}\left(p_{2}-p_{1}\right)_{\mu_{3}}+\eta_{\mu_{1} \mu_{3}}\left(p_{1}-p_{3}\right)_{\mu_{2}}+\eta_{\mu_{2} \mu_{3}}\left(p_{3}-p_{2}\right)_{\mu_{1}}\right],
\end{aligned}
$$

with $a^{\text {diam }}$ and $a^{\text {param }}$ as in Eq. (2.18). It is clear that the counterterm in Eq. (2.19) subtracts the UV divergences in the three-point function and that the beta function computed from the three-point function is also given by Eq. (2.21), all this in accordance with gauge invariance. We again see that the negative sign of the one-loop beta function is due to the dominance of the paramagnetic high-momentum contributions over the diamagnetic ones. We close this section displaying the leading non-commutative IR behaviour of the three-point function, obtained by summing the contributions in Eqs. (3.8) and (3.11):

$$
\begin{aligned}
& i \Gamma_{\mu_{1} \mu_{2} \mu_{3}}^{\mathrm{IR}}\left(p_{1}, p_{2}, p_{3}\right) \\
& =\frac{2}{\pi^{2}} \cos \left(\frac{p_{1} \wedge p_{2}}{2}\right) \sum_{i=1}^{3} \frac{\left(\tilde{p}_{i}\right)_{\mu_{1}}\left(\tilde{p}_{i}\right)_{\mu_{2}}\left(\tilde{p}_{i}\right)_{\mu_{3}}}{\left(\tilde{p}_{i}^{2}\right)^{2}} \\
& \quad+\frac{i}{3}\left(a^{\mathrm{diam}}+a^{\mathrm{param}}\right) 2 i \sin \left(\frac{p_{1} \wedge p_{2}}{2}\right)\left(\ln \tilde{p}_{1}^{2}+\ln \tilde{p}_{2}^{2}+\ln \tilde{p}_{3}^{2}\right) \\
& \quad \times\left[\eta_{\mu_{1} \mu_{2}}\left(p_{2}-p_{1}\right)_{\mu_{3}}+\eta_{\mu_{1} \mu_{3}}\left(p_{1}-p_{3}\right)_{\mu_{2}}+\eta_{\mu_{2} \mu_{3}}\left(p_{3}-p_{2}\right)_{\mu_{1}}\right] .
\end{aligned}
$$

Notice that the non-logarithmic non-commutative IR contributions to the three-point are only supplied by its diamagnetic part [see Eqs. (3.8) and (3.11)]; an effect that was met for the first time when we studied the vacuum polarization tensor.

\section{The four-point function}

In this section we compute the UV divergent and the leading non-commutative IR part of the four-point function and exhibit how UV/IR connects them. The four-point function 
$i \Gamma_{\mu_{1} \mu_{2} \mu_{3} \mu_{4}}\left(x_{1}, x_{2}, x_{3}, x_{4}\right)$ is defined by

$$
i \Gamma_{\mu_{1} \mu_{2} \mu_{3} \mu_{4}}\left(x_{1}, x_{2}, x_{3}, x_{4}\right)=\left.\frac{\delta^{4} \Gamma[B]}{\delta B_{\mu_{1}}\left(x_{1}\right) \delta B_{\mu_{2}}\left(x_{2}\right) \delta B_{\mu_{3}}\left(x_{3}\right) \delta B_{\mu_{4}}\left(x_{4}\right)}\right|_{B=0},
$$

where $i \Gamma[B]=\ln Z[B]$ and $Z[B]$ is as in Eq. (2.3). In momentum space we will write $i \Gamma_{\mu_{1} \mu_{2} \mu_{3} \mu_{4}}\left(p_{1}, p_{2}, p_{3}, p_{4}\right)$, with $p_{1}+p_{2}+p_{3}+p_{4}=0$, and will be the sum of a diamagnetic, a paramagnetic and a mixed contribution:

$$
\begin{aligned}
i \Gamma_{\mu_{1} \mu_{2} \mu_{3} \mu_{4}}\left(p_{1}, p_{2}, p_{3}, p_{4}\right)= & i \Gamma_{\mu_{1} \mu_{2} \mu_{3} \mu_{4}}^{\mathrm{diam}}\left(p_{1}, p_{2}, p_{3}, p_{4}\right) \\
& +i \Gamma_{\mu_{1} \mu_{2} \mu_{3} \mu_{4}}^{\mathrm{param}}\left(p_{1}, p_{2}, p_{3}, p_{4}\right) \\
& +i \Gamma_{\mu_{1} \mu_{2} \mu_{3} \mu_{4}}^{\mathrm{mixed}}\left(p_{1}, p_{2}, p_{3}, p_{4}\right) .
\end{aligned}
$$

Let us consider each one of these contributions separately.

The diamagnetic contribution is given by the sum of the diagrams in Fig. 7. The diagrams in the first, second and third row of the figure have different topologies and will be called box, lynx and bubble diagrams, respectively. Each one of these diagrams is a sum of a planar part and a non-planar part. The same arguments as in Sections 2 and 3 show that the planar parts are UV divergent by power counting at $D=4$, the divergence being logarithmic and being characterized as a pole in dimensional regularization. As regards
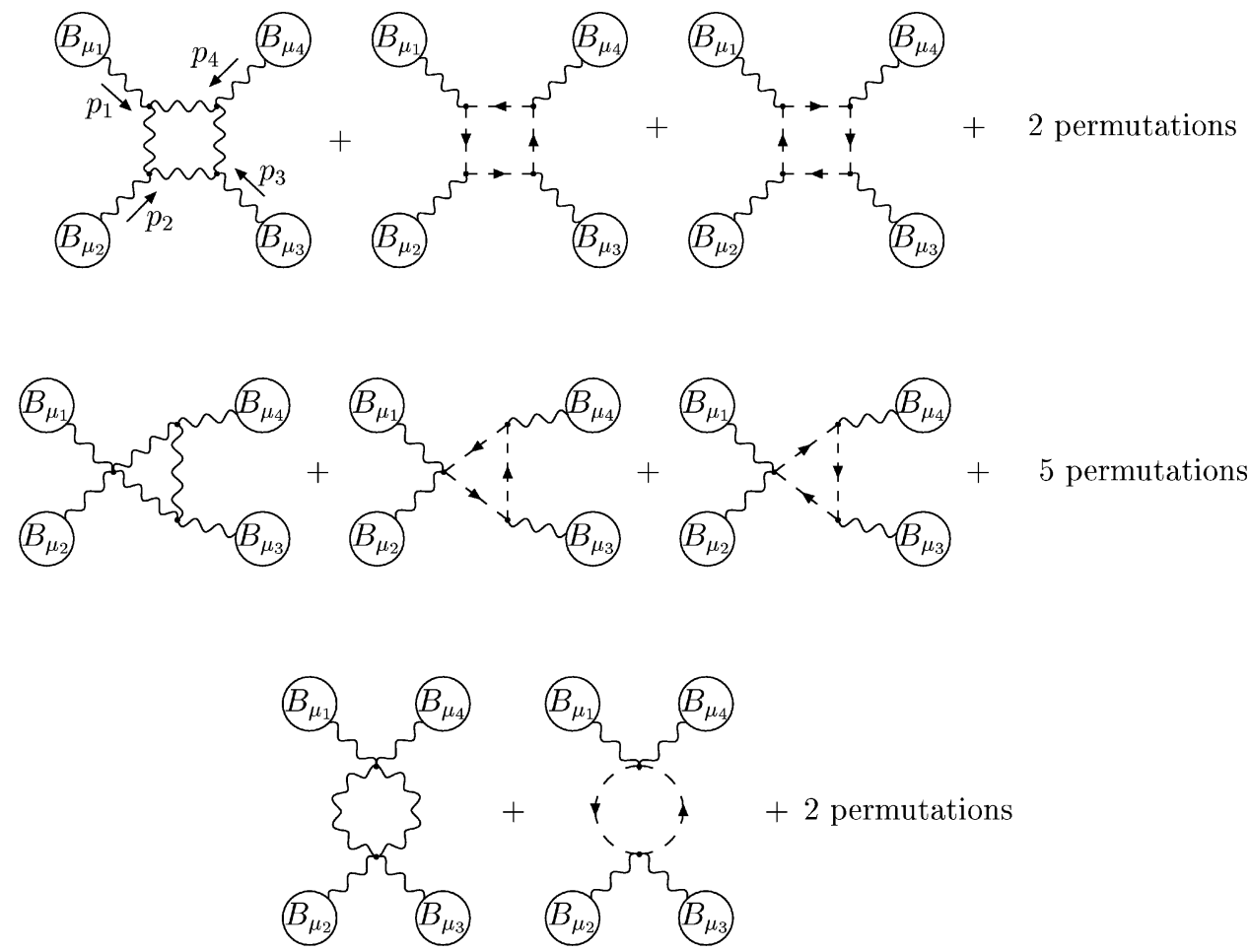

Fig. 7. One-loop 1PI diamagnetic diagrams contributing to the four-point function. 
the non-planar parts, these are finite for $\tilde{p}_{i} \neq 0$ and develop divergences for any of the configurations

$$
\begin{aligned}
& \tilde{p}_{1}=0, \quad \tilde{p}_{2}=0, \quad \tilde{p}_{3}=0, \quad \tilde{p}_{4}=0, \\
& \tilde{p}_{1}+\tilde{p}_{2}=0, \quad \tilde{p}_{1}+\tilde{p}_{3}=0, \quad \tilde{p}_{2}+\tilde{p}_{3}=0 .
\end{aligned}
$$

To calculate the UV divergences, we make a Laurent expansion about $D=4$ of the dimensionally regularized integrals defining the planar parts and obtain, for nonexceptional momenta:

$$
\begin{aligned}
& i \Gamma_{\mu_{1} \mu_{2} \mu_{3} \mu_{4}}^{\mathrm{box}, \mathrm{P}}\left(p_{1}, p_{2}, p_{3}, p_{4}\right) \\
& =\frac{-i}{16 \pi^{2} \varepsilon} \frac{8}{3}\left(\eta_{\mu_{1} \mu_{2}} \eta_{\mu_{3} \mu_{4}}+\eta_{\mu_{1} \mu_{3}} \eta_{\mu_{2} \mu_{4}}+\eta_{\mu_{1} \mu_{4}} \eta_{\mu_{2} \mu_{3}}\right) \\
& \quad \times\left[\cos \left(\frac{p_{1} \wedge p_{2}+p_{1} \wedge p_{3}+p_{2} \wedge p_{3}}{2}\right)\right. \\
& \quad+\cos \left(\frac{p_{1} \wedge p_{2}+p_{1} \wedge p_{3}-p_{2} \wedge p_{3}}{2}\right) \\
& \left.\quad+\cos \left(\frac{p_{1} \wedge p_{2}-p_{1} \wedge p_{3}-p_{2} \wedge p_{3}}{2}\right)\right]+O\left(\varepsilon^{0}\right)
\end{aligned}
$$

for the sums of the of the box diagrams, and

$$
\begin{aligned}
& i \Gamma_{\mu_{1} \mu_{2} \mu_{3} \mu_{4}}^{\text {lynx,P }}\left(p_{1}, p_{2}, p_{3}, p_{4}\right) \\
& =-\frac{1}{2} i \Gamma_{\mu_{1} \mu_{2} \mu_{3} \mu_{4}}^{\text {bub,P }}\left(p_{1}, p_{2}, p_{3}, p_{4}\right)+O\left(\varepsilon^{0}\right) \\
& =\frac{i}{16 \pi^{2} \varepsilon} 8\left[\left(\eta_{\mu_{1} \mu_{2}} \eta_{\mu_{3} \mu_{4}}+\eta_{\mu_{1} \mu_{4}} \eta_{\mu_{2} \mu_{3}}\right) \cos \left(\frac{p_{1} \wedge p_{2}+p_{1} \wedge p_{3}+p_{2} \wedge p_{3}}{2}\right)\right. \\
& \quad+\left(\eta_{\mu_{1} \mu_{3}} \eta_{\mu_{2} \mu_{4}}+\eta_{\mu_{1} \mu_{4}} \eta_{\mu_{2} \mu_{3}}\right) \cos \left(\frac{p_{1} \wedge p_{2}+p_{1} \wedge p_{3}-p_{2} \wedge p_{3}}{2}\right) \\
& \left.\quad+\left(\eta_{\mu_{1} \mu_{2}} \eta_{\mu_{3} \mu_{4}}+\eta_{\mu_{1} \mu_{3}} \eta_{\mu_{2} \mu_{4}}\right) \cos \left(\frac{p_{1} \wedge p_{2}-p_{1} \wedge p_{3}-p_{2} \wedge p_{3}}{2}\right)\right] \\
& \quad+O\left(\varepsilon^{0}\right)
\end{aligned}
$$

for the sum of the lynx and bubble diagrams. Here, as in previous sections, $D=4+2 \varepsilon$. Summing these three contributions and doing some simple trigonometry, we arrive at the following UV divergences for the diamagnetic part of the four-point function:

$$
\begin{aligned}
& i \Gamma_{\mu_{1} \mu_{2} \mu_{3} \mu_{4}}^{\mathrm{diam}, \mathrm{UV}}\left(p_{1}, p_{2}, p_{3}, p_{4}\right) \\
& =\frac{i a^{\mathrm{diam}}}{\varepsilon}\left[\left(\eta_{\mu_{1} \mu_{3}} \eta_{\mu_{2} \mu_{4}}-\eta_{\mu_{1} \mu_{4}} \eta_{\mu_{2} \mu_{3}}\right) \sin \left(\frac{p_{1} \wedge p_{2}}{2}\right) \sin \left(\frac{p_{3} \wedge p_{4}}{2}\right)\right. \\
& +\left(\eta_{\mu_{1} \mu_{3}} \eta_{\mu_{2} \mu_{4}}-\eta_{\mu_{1} \mu_{2}} \eta_{\mu_{3} \mu_{4}}\right) \sin \left(\frac{p_{1} \wedge p_{4}}{2}\right) \sin \left(\frac{p_{3} \wedge p_{2}}{2}\right) \\
& \left.+\left(\eta_{\mu_{1} \mu_{3}} \eta_{\mu_{2} \mu_{4}}-\eta_{\mu_{1} \mu_{2}} \eta_{\mu_{3} \mu_{4}}\right) \sin \left(\frac{p_{4} \wedge p_{2}}{2}\right) \sin \left(\frac{p_{3} \wedge p_{1}}{2}\right)\right],
\end{aligned}
$$


with $a^{\text {diam }}$ given in Eq. (2.18). Let us stress that, unlike for the three-point function, the trigonometric and tensor structure of the box, lynx and bubble contributions is different from that in the classical action $S_{\text {class }}[B]$ for the quartic vertex $B^{4}$, and that it is precisely the sum over diagrams with different topologies what produces the correct structure, thus making non-trivial the answer given by Eq. (4.5). Of course, this is so because gauge invariance is at work and brings the necessary simplifications.

We next compute the leading non-commutative IR behaviour of the non-planar parts of the diagrams in Fig. 7. To do this, we use Eqs. (3.5) and (3.6) and the result

$$
\begin{aligned}
& \int \frac{d^{4} q}{(2 \pi)^{4}} \frac{q_{\mu_{1}} q_{\mu_{2}} q_{\mu_{3}} q_{\mu_{4}} e^{i q \wedge k}}{q^{2}\left(q+\ell_{1}\right)^{2}\left(q+\ell_{2}\right)^{2}\left(q+\ell_{3}\right)^{2}} \\
& \approx \frac{-i}{16 \pi^{2}} \frac{1}{24} \ln \tilde{k}^{2}\left(\eta_{\mu_{1} \mu_{2}} \eta_{\mu_{3} \mu_{4}}+\eta_{\mu_{1} \mu_{3}} \eta_{\mu_{2} \mu_{4}}+\eta_{\mu_{1} \mu_{4}} \eta_{\mu_{2} \mu_{3}}\right)
\end{aligned}
$$

for $\tilde{k} \rightarrow 0$, where $\ell_{1}, \ell_{2}$ and $\ell_{3}$ never vanish and $\approx$ indicates that we have dropped contributions which remain finite as $\tilde{k} \rightarrow 0$. After some algebra, we obtain for the three sets of diagrams under consideration:

$$
\begin{aligned}
& i \Gamma_{\mu_{1} \mu_{2} \mu_{3} \mu_{4}}^{\text {box,NP }}\left(p_{1}, p_{2}, p_{3}, p_{4}\right) \frac{-i}{16 \pi^{2}}\left(\eta_{\mu_{1} \mu_{2}} \eta_{\mu_{3} \mu_{4}}+\eta_{\mu_{1} \mu_{3}} \eta_{\mu_{2} \mu_{4}}+\eta_{\mu_{1} \mu_{4}} \eta_{\mu_{2} \mu_{3}}\right) \\
& \times 4\left\{\cos \left(\frac{p_{1} \wedge p_{2}+p_{1} \wedge p_{3}+p_{2} \wedge p_{3}}{2}\right)\right. \\
& \times\left[\ln \left(\tilde{p}_{1}+\tilde{p}_{2}\right)^{2}+\ln \left(\tilde{p}_{2}+\tilde{p}_{3}\right)^{2}-\frac{2}{3} \sum_{i=1}^{4} \ln \tilde{p}_{i}^{2}\right] \\
&+\cos \left(\frac{p_{1} \wedge p_{2}+p_{1} \wedge p_{3}-p_{2} \wedge p_{3}}{2}\right) \\
& \times\left[\ln \left(\tilde{p}_{2}+\tilde{p}_{3}\right)^{2}+\ln \left(\tilde{p}_{1}+\tilde{p}_{3}\right)^{2}-\frac{2}{3} \sum_{i=1}^{4} \ln \tilde{p}_{i}^{2}\right] \\
&+\cos \left(\frac{p_{1} \wedge p_{2}+p_{1} \wedge p_{3}-p_{2} \wedge p_{3}}{2}\right) \\
&\left.\times\left[\ln \left(\tilde{p}_{1}+\tilde{p}_{2}\right)^{2}+\ln \left(\tilde{p}_{1}+\tilde{p}_{3}\right)^{2}-\frac{2}{3} \sum_{i=1}^{4} \ln \tilde{p}_{i}^{2}\right]\right\}
\end{aligned}
$$

and

$$
\begin{aligned}
& i \Gamma_{\mu_{1} \mu_{2} \mu_{3} \mu_{4}}^{\mathrm{lynx}, \mathrm{NP}}\left(p_{1}, p_{2}, p_{3}, p_{4}\right) \\
& \quad \approx-\frac{1}{2} i \Gamma_{\mu_{1} \mu_{2} \mu_{3} \mu_{4}}^{\mathrm{bub}, \mathrm{NP}}\left(p_{1}, p_{2}, p_{3}, p_{4}\right) \\
& \quad \approx \frac{-i}{16 \pi^{2}} 8\left\{\left[\left(\eta_{\mu_{1} \mu_{2}} \eta_{\mu_{3} \mu_{4}}+\eta_{\mu_{1} \mu_{4}} \eta_{\mu_{2} \mu_{3}}\right) \cos \left(\frac{p_{1} \wedge p_{2}+p_{1} \wedge p_{3}+p_{2} \wedge p_{3}}{2}\right)\right.\right.
\end{aligned}
$$




$$
\begin{aligned}
+ & \left(\eta_{\mu_{1} \mu_{2}} \eta_{\mu_{3} \mu_{4}}+\eta_{\mu_{1} \mu_{3}} \eta_{\mu_{2} \mu_{4}}\right) \cos \left(\frac{p_{1} \wedge p_{2}-p_{1} \wedge p_{3}-p_{2} \wedge p_{3}}{2}\right) \\
+ & \left.\left(\eta_{\mu_{1} \mu_{3}} \eta_{\mu_{2} \mu_{4}}+\eta_{\mu_{1} \mu_{4}} \eta_{\mu_{2} \mu_{3}}\right) \cos \left(\frac{p_{1} \wedge p_{2}+p_{1} \wedge p_{3}-p_{2} \wedge p_{3}}{2}\right)\right] \\
& \times \sum_{i=1}^{4} \ln \tilde{p}_{i}^{2} \\
- & {\left[\left(\eta_{\mu_{1} \mu_{2}} \eta_{\mu_{3} \mu_{4}}+2 \eta_{\mu_{1} \mu_{4}} \eta_{\mu_{2} \mu_{3}}\right) \cos \left(\frac{p_{1} \wedge p_{2}+p_{1} \wedge p_{3}+p_{2} \wedge p_{3}}{2}\right)\right.} \\
& \left.+\left(\eta_{\mu_{1} \mu_{2}} \eta_{\mu_{3} \mu_{4}}+2 \eta_{\mu_{1} \mu_{3}} \eta_{\mu_{2} \mu_{4}}\right) \cos \left(\frac{p_{1} \wedge p_{2}-p_{1} \wedge p_{3}-p_{2} \wedge p_{3}}{2}\right)\right] \\
& \left.\times \ln \left(\tilde{p}_{1}+\tilde{p}_{2}\right)^{2}\right) \\
- & {\left[\left(2 \eta_{\mu_{1} \mu_{2}} \eta_{\mu_{3} \mu_{4}}+\eta_{\mu_{1} \mu_{4}} \eta_{\mu_{2} \mu_{3}}\right) \cos \left(\frac{p_{1} \wedge p_{2}+p_{1} \wedge p_{3}+p_{2} \wedge p_{3}}{2}\right)\right.} \\
& \left.+\left(2 \eta_{\mu_{1} \mu_{2}} \eta_{\mu_{3} \mu_{4}}+\eta_{\mu_{1} \mu_{4}} \eta_{\mu_{2} \mu_{3}}\right) \cos \left(\frac{p_{1} \wedge p_{2}+p_{1} \wedge p_{3}-p_{2} \wedge p_{3}}{2}\right)\right] \\
& \left.\times \ln \left(\tilde{p}_{2}+\tilde{p}_{3}\right)^{2}\right) \\
- & {\left[\left(2 \eta_{\mu_{1} \mu_{2}} \eta_{\mu_{3} \mu_{4}}+\eta_{\mu_{1} \mu_{3}} \eta_{\mu_{2} \mu_{4}}\right) \cos \left(\frac{p_{1} \wedge p_{2}-p_{1} \wedge p_{3}-p_{2} \wedge p_{3}}{2}\right)\right.} \\
& \left.+\left(\eta_{\mu_{1} \mu_{3}} \eta_{\mu_{2} \mu_{4}}+2 \eta_{\mu_{1} \mu_{4}} \eta_{\mu_{2} \mu_{3}}\right) \cos \left(\frac{p_{1} \wedge p_{2}-p_{1} \wedge p_{3}-p_{2} \wedge p_{3}}{2}\right)\right] \\
& \left.\times \ln \left(\tilde{p}_{1}+\tilde{p}_{3}\right)^{2}\right\} .
\end{aligned}
$$

Summing these three results we obtain for the leading non-commutative IR diamagnetic contribution to the four-point function

$$
\begin{aligned}
i \Gamma_{\mu_{1} \mu_{2} \mu_{3} \mu_{4}}^{\operatorname{diam}, \mathrm{IR}}\left(p_{1}, p_{2}, p_{3}, p_{4}\right) & \\
=-4 i a^{\operatorname{diam}}\{ & \left(\eta_{\mu_{1} \mu_{3}} \eta_{\mu_{2} \mu_{4}}-\eta_{\mu_{1} \mu_{4}} \eta_{\mu_{2} \mu_{3}}\right) \sin \left(\frac{p_{1} \wedge p_{2}}{2}\right) \sin \left(\frac{p_{3} \wedge p_{4}}{2}\right) \\
& \times\left[\sum_{i=1}^{4} \ln \tilde{p}_{i}^{2}-3 \ln \left(\tilde{p}_{1}+\tilde{p}_{2}\right)^{2}\right] \\
+ & \left(\eta_{\mu_{1} \mu_{3}} \eta_{\mu_{2} \mu_{4}}-\eta_{\mu_{1} \mu_{2}} \eta_{\mu_{3} \mu_{4}}\right) \sin \left(\frac{p_{1} \wedge p_{4}}{2}\right) \sin \left(\frac{p_{3} \wedge p_{2}}{2}\right) \\
\times & {\left[\sum_{i=1}^{4} \ln \tilde{p}_{i}^{2}-3 \ln \left(\tilde{p}_{2}+\tilde{p}_{3}\right)^{2}\right] } \\
+ & \left(\eta_{\mu_{1} \mu_{3}} \eta_{\mu_{2} \mu_{4}}-\eta_{\mu_{1} \mu_{2}} \eta_{\mu_{3} \mu_{4}}\right) \sin \left(\frac{p_{4} \wedge p_{2}}{2}\right) \sin \left(\frac{p_{3} \wedge p_{1}}{2}\right) \\
\times & {\left.\left[\sum_{i=1}^{4} \ln \tilde{p}_{i}^{2}-3 \ln \left(\tilde{p}_{1}+\tilde{p}_{3}\right)^{2}\right]\right\}, }
\end{aligned}
$$


$a^{\text {diam }}$ being given in Eq. (2.18). Two comments are now in order. First, Eqs. (4.6) and (4.7) show that the non-planar parts of the four-point diamagnetic diagrams exhibit IR divergences for configurations (4.2). Yet, the full diamagnetic contribution, given by Eq. (4.8), is free of such singularities. There is, therefore, a cancellation mechanism at work among the non-commutative IR divergent contributions coming from the box, lynx and bubble diagrams. This mechanism is a consequence of gauge invariance being preserved at one-loop. Let us substantiate this statement. As we shall see in the next section, gauge invariance leads to the following Ward identity involving the three and four-point functions:

$$
\begin{aligned}
p_{4}^{\mu_{4}} \Gamma_{\mu_{1} \mu_{2} \mu_{3} \mu}\left(p_{1}, p_{2}, p_{3}, p_{4}\right) & \\
= & 2 \sin \left(\frac{p_{1} \wedge p_{4}}{2}\right) \Gamma_{\mu_{1} \mu_{2} \mu_{3}}\left(-p_{2}-p_{3}, p_{2}, p_{3}\right) \\
& +2 \sin \left(\frac{p_{2} \wedge p_{4}}{2}\right) \Gamma_{\mu_{1} \mu_{2} \mu_{3}}\left(-p_{1}-p_{3}, p_{3}, p_{1}\right) \\
& +2 \sin \left(\frac{p_{3} \wedge p_{4}}{2}\right) \Gamma_{\mu_{1} \mu_{2} \mu_{3}}\left(-p_{1}-p_{2}, p_{1}, p_{2}\right) .
\end{aligned}
$$

Now, the r.h.s of this equation is finite for any of the configurations (4.2), as can be readily shown by substituting Eq. (3.14) in it. Thus, if $\Gamma_{\mu_{1} \mu_{2} \mu_{3} \mu_{4}}\left(p_{1}, p_{2}, p_{3}, p_{4}\right)$ had an IR diamagnetic divergence for momenta (4.2), it would have to be transverse with respect to $p_{4}^{\mu_{4}}$. However, there is no tensor transverse to $p_{4}^{\mu_{4}}$ that can be built with the tensors and functions that occur in Eqs. (4.6) and (4.7). Secondly, the contribution to $i \Gamma_{\mu_{1} \mu_{2} \mu_{3} \mu_{4}}^{\text {diam,NP }}\left(p_{1}, p_{2}, p_{3}, p_{4}\right)$ coming from the momentum region

$$
\left|\tilde{p}_{1}\right| \sim\left|\tilde{p}_{2}\right| \sim\left|\tilde{p}_{3}\right| \sim\left|\tilde{p}_{4}\right| \sim\left|\tilde{p}_{1}+\tilde{p}_{2}\right| \sim\left|\tilde{p}_{1}+\tilde{p}_{3}\right| \sim\left|\tilde{p}_{2}+\tilde{p}_{3}\right| \sim \theta \Lambda_{\mathrm{IR}} \rightarrow 0
$$

is

$$
\begin{aligned}
& i \Gamma_{\mu_{1} \mu_{2} \mu_{3} \mu_{4}}^{\mathrm{diam}, \mathrm{NP}}\left(p_{1}, p_{2}, p_{3}, p_{4}\right) \\
& \approx-4 i a^{\operatorname{diam}} \ln \left(\theta \Lambda_{\mathrm{IR}}\right)^{2} \\
& \times\left[\left(\eta_{\mu_{1} \mu_{3}} \eta_{\mu_{2} \mu_{4}}-\eta_{\mu_{1} \mu_{4}} \eta_{\mu_{2} \mu_{3}}\right) \sin \left(\frac{p_{1} \wedge p_{2}}{2}\right) \sin \left(\frac{p_{3} \wedge p_{4}}{2}\right)\right. \\
& +\left(\eta_{\mu_{1} \mu_{3}} \eta_{\mu_{2} \mu_{4}}-\eta_{\mu_{1} \mu_{2}} \eta_{\mu_{3} \mu_{4}}\right) \sin \left(\frac{p_{1} \wedge p_{4}}{2}\right) \sin \left(\frac{p_{3} \wedge p_{2}}{2}\right) \\
& \left.+\left(\eta_{\mu_{1} \mu_{3}} \eta_{\mu_{2} \mu_{4}}-\eta_{\mu_{1} \mu_{2}} \eta_{\mu_{3} \mu_{4}}\right) \sin \left(\frac{p_{4} \wedge p_{2}}{2}\right) \sin \left(\frac{p_{3} \wedge p_{1}}{2}\right)\right] .
\end{aligned}
$$

As happened for the two-point and three-point functions, this logarithmic IR contribution can be retrieved from the UV divergent contribution $i \Gamma_{\mu_{1} \mu_{2} \mu_{3} \mu_{4}}^{\mathrm{diam}, \mathrm{UV}}\left(p_{1}, p_{2}, p_{3}, p_{4}\right)$ by performing the identifications (2.24). This provides an explicit realization for the four-point function of the UV/IR mixing characteristic of quantum field theories on non-commutative Minkowski spacetime. This UV/IR mixing works for each single Feynman diagram: note that one can apply the identifications (2.24) to Eqs. (4.3) and (4.4) to obtain Eqs. (4.6) and (4.7) for momenta verifying the conditions (4.10).

We now come to the paramagnetic part of the four-point function. It is given by the contribution quartic in the background field that comes from the diagram in Fig. 4, the 
latter being given by Eqs. (2.12) and (2.13). As already explained, the planar part of the diagram contains all UV divergences, while the non-planar part collects all integrals with non-commutative IR singularities. Projecting out from Eq. (2.12) the contribution quartic in $B_{\mu}$ and using the results of section 2 for $J(p)$, we obtain the following UV divergences and leading non-commutative IR terms in the paramagnetic contribution to the four-point function:

$$
\begin{aligned}
& i \Gamma_{\mu_{1} \mu_{2} \mu_{3} \mu_{4}}^{\text {param }}\left(p_{1}, p_{2}, p_{3}, p_{4}\right) \\
& =\frac{i a^{\text {param }}}{\varepsilon}[ \\
& \quad\left(\eta_{\mu_{1} \mu_{3}} \eta_{\mu_{2} \mu_{4}}-\eta_{\mu_{1} \mu_{4}} \eta_{\mu_{2} \mu_{3}}\right) \sin \left(\frac{p_{1} \wedge p_{2}}{2}\right) \sin \left(\frac{p_{3} \wedge p_{4}}{2}\right) \\
& \quad+\left(\eta_{\mu_{1} \mu_{3}} \eta_{\mu_{2} \mu_{4}}-\eta_{\mu_{1} \mu_{2}} \eta_{\mu_{3} \mu_{4}}\right) \sin \left(\frac{p_{1} \wedge p_{4}}{2}\right) \sin \left(\frac{p_{3} \wedge p_{2}}{2}\right) \\
& \left.\quad+\left(\eta_{\mu_{1} \mu_{3}} \eta_{\mu_{2} \mu_{4}}-\eta_{\mu_{1} \mu_{2}} \eta_{\mu_{3} \mu_{4}}\right) \sin \left(\frac{p_{4} \wedge p_{2}}{2}\right) \sin \left(\frac{p_{3} \wedge p_{1}}{2}\right)\right]
\end{aligned}
$$

and

$$
\begin{aligned}
& i \Gamma_{\mu_{1} \mu_{2} \mu_{3} \mu_{4}}^{\mathrm{param}, \mathrm{R}}\left(p_{1}, p_{2}, p_{3}, p_{4}\right) \\
& =-4 i a^{\operatorname{param}}\left[\left(\eta_{\mu_{1} \mu_{3}} \eta_{\mu_{2} \mu_{4}}-\eta_{\mu_{1} \mu_{4}} \eta_{\mu_{2} \mu_{3}}\right) \sin \left(\frac{p_{1} \wedge p_{2}}{2}\right) \sin \left(\frac{p_{3} \wedge p_{4}}{2}\right)\right. \\
& \times \ln \left(\tilde{p}_{1}+\tilde{p}_{2}\right)^{2} \\
& +\left(\eta_{\mu_{1} \mu_{3}} \eta_{\mu_{2} \mu_{4}}-\eta_{\mu_{1} \mu_{2}} \eta_{\mu_{3} \mu_{4}}\right) \sin \left(\frac{p_{1} \wedge p_{4}}{2}\right) \sin \left(\frac{p_{3} \wedge p_{2}}{2}\right) \\
& \times \ln \left(\tilde{p}_{2}+\tilde{p}_{3}\right)^{2} \\
& +\left(\eta_{\mu_{1} \mu_{3}} \eta_{\mu_{2} \mu_{4}}-\eta_{\mu_{1} \mu_{2}} \eta_{\mu_{3} \mu_{4}}\right) \sin \left(\frac{p_{4} \wedge p_{2}}{2}\right) \sin \left(\frac{p_{3} \wedge p_{1}}{2}\right) \\
& \left.\times \ln \left(\tilde{p}_{1}+\tilde{p}_{3}\right)^{2}\right],
\end{aligned}
$$

$a^{\text {param }}$ being as in Eq. (2.18). Note that, despite the fact that the paramagnetic contribution to the four-point function is made of integrals $J(p)$, with $p=p_{1}+p_{2}, p_{2}+p_{3}, p_{1}+p_{3}$, whose non-planar parts become singular for $\tilde{p}=0, i \Gamma_{\mu_{1} \mu_{2} \mu_{3} \mu_{4}}^{\mathrm{param}, \mathrm{IR}}\left(p_{1}, p_{2}, p_{3}, p_{4}\right)$ is finite for any configuration (4.2). This finiteness can be explained as a consequence of gauge invariance following the same lines as for the diamagnetic contribution: see paragraph below Eq. (4.8). Also note that in the momentum region (4.10) the value of $i \Gamma_{\mu_{1} \mu_{2} \mu_{3} \mu_{4}}^{\text {param,IR }}\left(p_{1}, p_{2}, p_{3}, p_{4}\right)$ can be obtained from the UV divergent contribution $i \Gamma_{\mu_{1} \mu_{2} \mu_{3} \mu_{4}}^{\mathrm{param}, \mathrm{UV}}\left(p_{1}, p_{2}, p_{3}, p_{4}\right)$ by using the identifications (2.24), in accordance with UV/IR mixing.

It remains to study the mixed contribution to the four-point function. There are quite a few diagrams producing contributions quartic in the background field with both diamagnetic and paramagnetic vertices. It is straightforward to see, however, that their planar parts are all UV finite by power counting and that their non-planar parts are free of singularities for momenta (4.2), so we conclude that

$$
\Gamma_{\mu_{1} \mu_{2} \mu_{3} \mu_{4}}^{\text {mixe,UV }}\left(p_{1}, p_{2}, p_{3}, p_{4}\right)=\Gamma_{\mu_{1} \mu_{2} \mu_{3} \mu_{4}}^{\text {mixed,IR }}\left(p_{1}, p_{2}, p_{3}, p_{4}\right)=0 .
$$


We are now ready to give the complete UV divergent contribution $i \Gamma_{\mu_{1} \mu_{2} \mu_{3} \mu_{4}}^{\mathrm{UV}}\left(p_{1}, p_{2}, p_{3}, p_{4}\right)$ to the four-point function. It is the sum of the $\mathrm{UV}$ divergent diamagnetic and paramagnetic contributions and takes the form

$$
\begin{aligned}
& i \Gamma_{\mu_{1} \mu_{2} \mu_{3} \mu_{4}}^{\mathrm{UV}}\left(p_{1}, p_{2}, p_{3}, p_{4}\right) \\
& =\frac{i}{\varepsilon}\left(a^{\text {diam }}+a^{\text {param }}\right) \\
& \times\left[\left(\eta_{\mu_{1} \mu_{3}} \eta_{\mu_{2} \mu_{4}}-\eta_{\mu_{1} \mu_{4}} \eta_{\mu_{2} \mu_{3}}\right) \sin \left(\frac{p_{1} \wedge p_{2}}{2}\right) \sin \left(\frac{p_{3} \wedge p_{4}}{2}\right)\right. \\
& +\left(\eta_{\mu_{1} \mu_{3}} \eta_{\mu_{2} \mu_{4}}-\eta_{\mu_{1} \mu_{2}} \eta_{\mu_{3} \mu_{4}}\right) \sin \left(\frac{p_{1} \wedge p_{4}}{2}\right) \sin \left(\frac{p_{3} \wedge p_{2}}{2}\right) \\
& \left.+\left(\eta_{\mu_{1} \mu_{3}} \eta_{\mu_{2} \mu_{4}}-\eta_{\mu_{1} \mu_{2}} \eta_{\mu_{3} \mu_{4}}\right) \sin \left(\frac{p_{4} \wedge p_{2}}{2}\right) \sin \left(\frac{p_{3} \wedge p_{1}}{2}\right)\right] \text {. }
\end{aligned}
$$

It follows that the MS counterterm (2.19) subtracts the UV divergences in the four-point function and that the beta function computed from the resulting renormalized four-point is as in Eq. (2.21). As concerns the leading non-commutative IR contribution to the four-point function, it is finite after summing over diagrams and is given by the sum of Eqs. (4.8) and (4.13):

$$
\begin{aligned}
i \Gamma_{\mu_{1} \mu_{2} \mu_{3} \mu_{4}}^{\mathrm{IR}}\left(p_{1}, p_{2}, p_{3}, p_{4}\right)= & \left.i \Gamma_{\mu_{1} \mu_{2} \mu_{3} \mu_{4}}^{\mathrm{diam}, p_{1}}, p_{2}, p_{3}, p_{4}\right) \\
& +i \Gamma_{\mu_{1} \mu_{2} \mu_{3} \mu_{4}}^{\mathrm{param}, \mathrm{IR}}\left(p_{1}, p_{2}, p_{3}, p_{4}\right) .
\end{aligned}
$$

\section{Gauge invariance}

In this section we shall prove that gauge invariance for the one-loop renormalized theory as mathematically expressed by the equation

$$
\int d^{D} x \omega(x) D_{\mu}[B] \frac{\delta \Gamma[B]}{\delta B_{\mu}(x)}=0
$$

holds, where $i \Gamma[B]=\ln Z[B]$ and $Z[B]$ is given in Eq. (2.3). In this regard it is worth noting that, to the best of our knowledge, it remains an open question [20] whether nonlogarithmic non-commutative IR divergences are compatible with gauge invariance and finiteness. Here we see that non-logarithmic IR divergences preserve gauge invariance.

We have shown in Sections 2, 3 and 4 that, to subtract the UV divergences in the two, three and four-point functions, it is enough to add the counterterm (2.19)

$$
\delta S[B]=\frac{1}{4 \varepsilon}\left(a^{\text {diam }}+a^{\text {param }}\right) \int d^{D} x F^{\mu v}[B] \star F_{\mu \nu}[B] .
$$

This counterterm satisfies Eq. (5.1) and does not change the non-commutative IR behaviour of the Green functions. Hence, the renormalized two, three and four-point functions have the same non-commutative IR behaviour as the regularized ones, given in Eqs. (2.25), (3.14) and (4.16). We must check that these expressions are consistent with the Ward 
identity (5.1). To this end, we note that Eq. (5.1) implies

$$
\begin{aligned}
& p^{v} \Pi_{\mu v}(p)=0, \\
& p_{3}^{\mu_{3}} \Gamma_{\mu_{1} \mu_{2} \mu_{3}}\left(p_{1}, p_{2}, p_{3}\right)=2 i \sin \left(\frac{p_{1} \wedge p_{2}}{2}\right)\left[\Pi_{\mu_{1} \mu_{2}}\left(p_{1}\right)-\Pi_{\mu_{1} \mu_{2}}\left(p_{2}\right)\right],
\end{aligned}
$$

together with Eq. (4.9). It is apparent that the non-commutative IR divergent contribution $\Pi_{\mu \nu}^{\mathrm{IR}}(p)$ to the vacuum polarization tensor in Eq. (2.25) verifies the identity (5.3). It is also clear that, neglecting terms which remain finite for some $i(i=1,2,3)$ in the limit $\tilde{p}_{i} \rightarrow 0$, we have

$$
\begin{aligned}
p_{3}^{\mu_{3}} \Gamma_{\mu_{1} \mu_{2} \mu_{3}}^{\mathrm{IR}}\left(p_{1}, p_{2}, p_{3}\right) & \approx-\frac{2}{\pi^{2}}\left(p_{1} \wedge p_{2}\right)\left[\frac{\left(\tilde{p}_{1}\right)_{\mu_{1}}\left(\tilde{p}_{1}\right)_{\mu_{2}}}{\tilde{p}_{1}^{2}}-\frac{\left(\tilde{p}_{2}\right)_{\mu_{1}}\left(\tilde{p}_{2}\right)_{\mu_{2}}}{\tilde{p}_{2}^{2}}\right] \\
& \approx 2 i \sin \left(\frac{p_{1} \wedge p_{2}}{2}\right)\left[\Pi_{\mu_{1} \mu_{2}}^{\mathrm{IR}}\left(p_{1}\right)-\Pi_{\mu_{1} \mu_{2}}^{\mathrm{IR}}\left(p_{2}\right)\right],
\end{aligned}
$$

in agreement with the Ward identity (5.4) above. As concerns the leading non-commutative IR behaviour of the four-point function, its consistency with gauge invariance has already been thoroughly checked in Section 4.

We end this section writing the effective action which reproduces the logarithmic contributions in the non-commutative IR momentum regions (2.23), (3.9) and (4.10) to the vacuum polarization tensor, three-point function and four-point function. From the results derived in Sections 2, 3 and 4 it is clear that it has the form

$$
\frac{1}{4}\left(a^{\text {diam }}+a^{\text {param }}\right) \ln \left(\theta \Lambda_{\mathrm{IR}}\right)^{2} \int d^{4} x\left(F^{\mu \nu}[B] \star F_{\mu \nu}[B]\right),
$$

with the already known result (2.18) for $a^{\text {diam }}$ and $a^{\text {param }}$. In accordance with Ref. [10], it is dual to the UV counterterm (5.2) under the identifications (2.24).

\section{Conclusions and suggestions}

In this paper we have computed the complete UV and non-commutative IR divergent contributions to the one-loop effective action of $U(1)$ gauge theory on non-commutative Minkowski spacetime in a background field. The UV divergences arise from the planar parts of the one-loop diagrams, and come from virtual quanta with arbitrarily high momenta moving around the loop of the diagrams. The non-commutative IR divergent contributions are also produced by high-momentum virtual quanta, but occur in the nonplanar parts of the diagrams. The vacuum polarization has quadratic and logarithmic noncommutative IR divergent contributions, while the three-point function has linear and logarithmic contributions, and the four-point function only has logarithmic contributions $[11,12]$. Logarithmic contributions add to an overall IR finite contribution for the three and four-point functions, while this is not so for the vacuum polarization tensor. The overall logarithmic non-commutative IR behaviour of the three Green functions is dual to the overall UV divergent behaviour in the sense that the identifications (2.24) transform one into another. 
The main result presented in this article is the following. We have shown that the paramagnetic contributions to the effective action dominate over the diamagnetic contributions at very high momentum. Here we say that we are in the high-momentum or high-energy region if the momentum is large as measured against the non-commutative energy scale $|\theta|^{-1 / 2}$. It turns out that, in the high-momentum regime, paramagnetic contributions give rise to anti-screening of the coupling constant, whereas diamagnetic contributions produce screening. The combined effect explains the negative sign of the beta function and its actual value. We recall that, while for a Lorentz invariant theory paramagnetism always comes with charge anti-screening and diamagnetism with charge screening [2], this is not necessarily so if the theory lacks Lorentz invariance, a circumstance that occurs if space is non-commutative. Yet the net result is analogous to that for Yang-Mills theory on commutative Minkowski spacetime. The reason for this is that the beta function can be computed from the UV divergences in the vacuum polarization tensor, provided gauge invariance holds. These divergences occurring only in the nonplanar part implies that they are independent of $\theta^{\mu \nu}$ and do not break Lorentz invariance.

We have also shown that the quadratic and linear non-commutative IR divergences that exhibit the vacuum polarization tensor and three-point function have a purely diamagnetic origin. By contrast, the leading non-commutative IR logarithmic contributions to the vacuum polarization tensor, the three-point and the four-point functions receive both paramagnetic an diamagnetic contributions. The duality of logarithmic contributions to UV divergences discussed above does not need to sum over diamagnetic and paramagnetic contributions but holds for each of them separately.

Let us now look at the renormalized theory and consider the non-commutative IR regime (4.10). As shown in Section 5, the leading non-commutative IR logarithmic contribution to the effective action in this region has the form (5.5). This contribution prevails over logarithmic contributions $\ln \left(p^{2} / \mu^{2}\right)$, if $p^{2}$ is of the same order of magnitude as the squared renormalization scale $\mu^{2}$. This leads us to claim that, in this regime, Eq. (5.5) can be thought of as giving rise to an effective IR renormalization of the renormalized coupling constant of the theory $g^{2}(\mu)$, thus introducing an IR effective coupling $g_{\mathrm{IR}}^{2}$ given by

$$
\frac{1}{g_{\mathrm{IR}}^{2}}=\frac{1}{g^{2}(\mu)}-\left(a^{\mathrm{diam}}+a^{\mathrm{param}}\right) \ln \left(\theta \Lambda_{\mathrm{IR}} \mu\right)^{2},
$$

with $a^{\text {diam }}$ and $a^{\text {param }}$ as in Eq. (2.18). In other words, in the non-commutative IR domain (4.10), the dominant logarithmic contributions to the 1PI one-loop Green functions of the theory are given by their tree-level expressions at finite $\theta$ but with $g^{2}(\mu)$ replaced with $g_{\mathrm{IR}}^{2}$. Note also that, in this non-commutative IR region, the paramagnetic contributions produce a screening of the charge, whereas the diamagnetic contributions anti-screen the charge. This does not come as a surprise since we know that when Lorentz invariance is lost there are media where paramagnetism comes with screening and diamagnetism with anti-screening [21], the speed of light being not equal to 1 . This is precisely what happens in the case at hand. As pointed out in Ref. [12], the quadratic non-commutative IR divergent behaviour of the vacuum polarization tensor gives rise to a modification of the dispersion relation for photon polarizations parallel to $\tilde{p}^{\mu}$. Indeed, let $p^{\mu}=(E, \vec{p})$, with 
$\vec{p}=\left(p^{1}, p^{2}, p^{3}\right)$, and take a photon polarized along the direction defined by $\tilde{p}^{\mu}$. We then have

$$
E^{2}=\left(p^{3}\right)^{2}+\vec{P}^{2}\left(1-\frac{2}{\pi^{2}} \frac{g^{2}}{\theta^{2}|\vec{P}|^{4}}\right),
$$

where $\vec{P}=\left(p^{1}, p^{2}, 0\right)$. This equation leads to a photon speed $\vec{v}_{g}=\vec{\nabla}_{\vec{p}} E$, with modulus greater than 1 in the region $2 g^{2} \ll \pi^{2} \theta^{2}|\vec{P}|^{4}$ where perturbation theory is valid. Whether this signals a true instability of the theory or is an artifact of perturbation theory remains an open question $[22,23]$.

One may wonder if the results presented here for $U(1)$ gauge theory generalize to the $U(N)$ case. In this regard we note that, although in the non-commutative IR domain no new divergent dependence on the external momenta appears, the colour structures of the UV and the non-commutative IR divergent parts are different $[9,10]$. This indicates that UV/IR duality in this case is more involved and deserves further investigation. Finally, it will be worth exploring whether there exists a connection between the results presented here and the dipole structures that occur in non-commutative quantum field theories [24].

\section{Acknowledgement}

The authors are grateful to E. López for pointing them out that the relative sign in the dispersion relation (6.1) in a previous version of this paper was wrong. They also thank CICyT, Spain for financial support through grant No. PB98-0842.

\section{References}

[1] G. 't Hooft (unpublished), as quoted in, P.D. Mannheim, Phys. Rev. D 9 (1974) 3438; N.K. Nielsen, Am. J. Phys. 49 (12) (1981) 1171.

[2] R.J. Hughes, Phys. Lett. B 97 (1981) 246; R.J. Hughes, Nucl. Phys. B 186 (1981) 376.

[3] A.M. Polyakov, Gauge Fields and Strings, Harwood Academic, 1987.

[4] L.F. Abott, Nucl. Phys. B 185 (1981) 189.

[5] E. Álvarez, C. Gómez, JHEP 9910 (1999) 018.

[6] P. Di Vecchia, L. Magnea, A. Lerda, R. Russo, R. Marotta, Nuc. Phys. B 469 (1996) 235.

[7] T. Krajewski, R. Wulkenhaar, J. Mod. Phys. A 15 (2000) 1011; C.P. Martín, D. Sánchez-Ruiz, Phys. Rev. Lett. 83 (1999) 476.

[8] M. Sheikh-Jabbari, JHEP 9906 (1999) 015.

[9] A. Armoni, Comments on perturbative dynamics of non-commutative Yang-Mills theory, hepth/0005208.

[10] S. Minwalla, M.V. Raamsdonk, N. Seiberg, JHEP 0002 (2000) 020.

[11] M. Hayakawa, Phys. Lett. B 478 (2000) 394.

[12] A. Matusis, L. Susskind, N. Toumbas, The IR/UV connection in the non-commutative gauge theories, hep-th/0002075.

[13] J. Ambjorn, Y.M. Makeenko, J. Nishimura, R.J. Szabo, Lattice gauge fields and discrete noncommutative Yang-Mills theory, hep-th/0004147. 
[14] O. Andreev, H. Dorn, Diagrams of noncommutative phi-three theory from string theory, hep-th/ 0003113 ;

A. Bilal, C.-S. Chu, R. Russo, String theory and noncommutative field theories at one oop, hep-th/0003180;

Y. Kiem, S. Lie, UV/IR mixing in noncommutative field theory via open string loops, hep-th/ 0003145 ;

H. Liu, J. Michelson, Stretched strings in noncommutative field theory, hep-th/0004013.

[15] J. Gomis, M. Kleban, T. Mehen, M. Rangamani, S. Shenker, Noncommutative gauge dynamics from the string worldsheet, hep-th/0003215.

[16] N. Seiberg, L. Susskind, N. Toumbas, Strings in background electric field, space/time noncommutativity and a new noncritical string theory, hep-th/0005040;

J.L.F. Barbón, E. Rabinovici, Stringy fuzziness as the custodian of time-space noncommutativity, hep-th/0005073.

[17] J. Gomis, T. Mehen, Spacetime noncommutative field theories and unitarity, hep-th/0005129.

[18] E.R. Speer, Generalized Feynman amplitudes, Princeton Univ. Press, 1969.

[19] N. Seiberg, E. Witten, JHEP 9909 (1999) 032.

[20] I. Chepelev, R. Roiban, JHEP 0005 (2000) 037;

A.A. Bichl, J.M. Grimstrup, H. Grosse, L. Popp, M. Schweda, R. Wulkenhaar, The superfield formalism applied to the noncommutative Wess-Zumino model, hep-th/0007050.

[21] J. Ziman, Principles of the theory of solids, Cambridge Univ. Press, 1979.

[22] J. Gomis, T. Mehen, M. Wise, Quantum field theories with compact noncommutative extra dimensions, hep-th/0006160.

[23] K. Landsteiner, E. López, M.H.G. Tytgat, JHEP 0009 (2000) 027.

[24] C.-S. Chu, P.-M. Ho, Nucl. Phys. B 550 (1999) 151;

M.M. Sheikh-Jabbary, Phys. Lett. B 455 (1999) 129;

D. Bigatti, L. Susskind, Magnetic fields, branes and noncommutative geometry, hep-th/ 9908056;

Z. Yin, Phys. Lett. B 466 (1999) 234;

L. Alvarez-Gaumé, J.L.F. Barbón, Non-linear vacuum phenomena in non-commutative QED, hep-th/0006209. 